\title{
Rhodium Catalysed Intermolecular Chelation Controlled Alkene and Alkyne Hydroacylation: Synthetic Scope of $\beta-S$-Substituted Aldehyde Substrates
}

Michael C. Willis, ${ }^{\dagger *}$ Helen E. Randell-Sly, ${ }^{\dagger}$ Robert L. Woodward, ${ }^{\dagger}$ Steven J. McNally ${ }^{\dagger}$ and Gordon S. Currie

${ }^{\dagger}$ Department of Chemistry, University of Bath, Bath, BA2 7AY, UK. ${ }^{\ddagger}$ AstraZeneca, Alderley Park, Macclesfield, Cheshire, SK10 4TG, UK

\section{Supporting Information}

\section{Contents}

\begin{tabular}{|c|c|c|c|}
\hline Page & & Page & \\
\hline $\mathrm{S} 1$ & Contents & $\mathrm{S} 28$ & Table 5 entry $5{ }^{1} \mathrm{H}$ NMR \\
\hline $\mathrm{S} 2$ & $\begin{array}{l}\text { General comments; Table } 3 \text { entry } 1 \\
\text { substrate; Table } 3 \text { entry } 1\end{array}$ & $\mathrm{~S} 29$ & Table 5 entry $6{ }^{1} \mathrm{H}$ NMR \\
\hline S3 & Table 3 entry 2 substrate; Table 3 entry 2 & $\mathrm{~S} 30$ & Table 5 entry $7{ }^{1} \mathrm{H}$ NMR \\
\hline S4 & $\begin{array}{l}\text { Table } 3 \text { entry } 3 \text { substrate; Table } 3 \text { entry } 3 \text {; } \\
\text { Table } 3 \text { entry } 4 \text { substrate }\end{array}$ & $\mathrm{S} 31$ & Table 5 entry $8{ }^{1} \mathrm{H}$ NMR \\
\hline S5 & $\begin{array}{l}\text { Table } 3 \text { entry } 4 \text {; Table } 3 \text { entry } 5 \text { substrate; } \\
\text { Table } 3 \text { entry } 5\end{array}$ & $\mathrm{~S} 32$ & Table 5 entry $9{ }^{1} \mathrm{H}$ NMR \\
\hline S6 & Table 3 entry 6 substrate; Table 3 entry 6 & $\mathrm{~S} 33$ & Table 5 entry $10{ }^{1} \mathrm{H}$ NMR \\
\hline S7 & 4; Table 5 entry 1 & $\mathrm{~S} 34$ & Table 5 entry $11{ }^{1} \mathrm{H}$ NMR \\
\hline S8 & Table 5 entry 3; Table 5 entry 5 & $\mathrm{~S} 35$ & Table 5 entry $12{ }^{1} \mathrm{H}$ NMR \\
\hline S9 & Table 5 entry 6 ; Table 5 entry 7 & $\mathrm{~S} 36$ & Table 5 entry $13{ }^{1} \mathrm{H}$ NMR \\
\hline $\mathrm{S} 10$ & Table 5 entry 8; Table 5 entry 9 & $\mathrm{~S} 37$ & Table 5 entry $14{ }^{1} \mathrm{H}$ NMR \\
\hline $\mathrm{S} 11$ & Table 5 entry 10 ; Table 5 entry 11 ; & $\mathrm{S} 38$ & Table 5 entry $15{ }^{1} \mathrm{H}$ NMR \\
\hline $\mathrm{S} 12$ & Table 5 entry 12; Table 5 entry 13; & $\mathrm{S} 39$ & Table 5 entry $17{ }^{1} \mathrm{H}$ NMR \\
\hline S13 & Table 5 entry 14; Table 5 entry 15 ; & $\mathrm{S} 40$ & $6{ }^{1} \mathrm{H}$ NMR \\
\hline S14 & Table 5 entry $17 ; 6$ & S41 & $7{ }^{1} \mathrm{H}$ NMR \\
\hline S15 & 7 ; Table 6 entry 1 & $\mathrm{~S} 42$ & Table 6 entry $1{ }^{1} \mathrm{H}$ NMR \\
\hline S16 & Table 6 entry 2; & $\mathrm{S} 43$ & Table 6 entry $1{ }^{1} \mathrm{H}$ NMR \\
\hline S17 & Table 6 entry 3; Table 6 entry 4 substrate & $\mathrm{S} 44$ & Table 6 entry $2{ }^{1} \mathrm{H}$ NMR \\
\hline $\mathrm{S} 18$ & Table 6 entry 4 & $\mathrm{~S} 45$ & Table 6 entry $2{ }^{1} \mathrm{H}$ NMR \\
\hline S19 & Table 3 entry $1{ }^{1} \mathrm{H}$ NMR & S46 & Table 6 entry $3{ }^{1} \mathrm{H}$ NMR \\
\hline S20 & Table 3 entry $2{ }^{1} \mathrm{H}$ NMR & S47 & $\begin{array}{l}\text { Table } 6 \text { entry } 4 \text { substrate } \\
{ }^{1} \mathrm{H} \text { NMR }\end{array}$ \\
\hline S21 & Table 3 entry $3{ }^{1} \mathrm{H}$ NMR & $\mathrm{S} 48$ & Table 6 entry $4{ }^{1} \mathrm{H}$ NMR \\
\hline S22 & Table 3 entry $4{ }^{1} \mathrm{H}$ NMR & $\mathrm{S} 49$ & Table 6 entry $4{ }^{1} \mathrm{H}$ NMR \\
\hline S23 & Table 3 entry $5{ }^{1} \mathrm{H}$ NMR & & \\
\hline $\mathrm{S} 24$ & Table 3 entry $6{ }^{1} \mathrm{H}$ NMR & & \\
\hline $\mathrm{S} 25$ & $4{ }^{1} \mathrm{H}$ NMR & & \\
\hline S26 & Table 5 entry $1{ }^{1} \mathrm{H}$ NMR & & \\
\hline S27 & Table 5 entry $3{ }^{1} \mathrm{H}$ NMR & & \\
\hline
\end{tabular}




\section{General}

IR spectra were recorded using $\mathrm{NaCl}$ discs. ${ }^{1} \mathrm{H}$ NMR spectra were obtained at 300 $\mathrm{MHz}$, unless otherwise noted, with tetramethylsilane as an internal standard. $J$ values are given in Hz. ${ }^{13} \mathrm{C}$ NMR spectra were obtained at $75 \mathrm{MHz}$, unless otherwise noted. All dry solvents were freshly distilled under nitrogen prior to use. Acetone was distilled from Drierite and stored over $4 \AA$ molecular sieves. Petrol refers to the fraction of petroleum ether obtained between $40-60{ }^{\circ} \mathrm{C}$. All glassware was dried in an oven and allowed to cool under nitrogen prior to use. All reactions were carried out under argon unless otherwise stated. All commercial reagents were used as obtained. Flash chromatography was conducted under medium pressure, using matrix 60 silica.

General procedure for aldehyde reactions as exemplified by 3-ethanesulfanylpropionaldehyde, Table 3 entry 1 substrate<smiles>CCSCCC=O</smiles>

Following the general procedure as described by Weber, ${ }^{1}$ employing sodium hydride in place of sodium. In a representative example; to a neat solution of ethane thiol (13.0 g, $226 \mathrm{mmol})$ and acrolein $(10.0 \mathrm{~g}, 178 \mathrm{mmol})$ was added $\mathrm{NaH}(36 \mathrm{mg}, 0.89$ mmol), cautiously over 30 minutes, (care! extremely exothermic reaction ensues). The resulting mixture was stirred at room temperature for 16 hours before removal of volatiles in vacuo and subsequent short path distillation under reduced pressure afforded the aldehyde as a colorless oil, which was used directly in subsequent hydroacylation reactions $(14 \mathrm{~g}, 62 \%) ; \delta_{\mathrm{H}}\left(300 \mathrm{MHz}, \mathrm{CDCl}_{3}\right) 9.72(1 \mathrm{H}, \mathrm{s}), 2.78-2.72$ (2 H, m), 2.70-2.62 (2 H, m), 2.50 (2 H, q, J 7.6), 1.20 (3 H, t, J 7.6).

General procedure for hydroacylation reactions as exemplified by methyl 6ethanesulfanyl-4-oxo-hexanoate, Table 3 entry 1<smiles>CCCCC(=O)CCC(=O)OC</smiles>

Acetone $(2.0 \mathrm{~mL})$ was added under argon to pre-catalyst $[\mathrm{Rh}(\mathrm{nbd})(\mathrm{dppe})] \mathrm{ClO}_{4}(10$ $\mathrm{mg}, 0.015 \mathrm{mmol})$. The catalyst was activated in situ by bubbling though the solution with $\mathrm{H}_{2}$ for 2 minutes untill a colour change from orange to yellow was observed.

\footnotetext{
${ }^{1}$ Weber, H. DE855704, 1952.
} 
After this time the solution was purged with argon. To this solution 3(ethanesulfanyl)propionaldehyde (17 $\mu \mathrm{L}, 0.15 \mathrm{mmol}$ ) was added followed by methyacrylate $(65 \mu \mathrm{L}, 0.7 \mathrm{mmol})$. The resulting mixture was stirred and heated at 55 ${ }^{\circ} \mathrm{C}$ for 16 hours. After this time the solution was reduced in vacu and purified by flash chromatography ( 1:4 EtOAc:Petrol) to give title compound as a colourless oil (20 mg, $68 \%) ; \delta_{\mathrm{H}}\left(300 \mathrm{MHz}, \mathrm{CDCl}_{3}\right) 3.61(3 \mathrm{H}, \mathrm{s}), 2.71-2.65(4 \mathrm{H}, \mathrm{m}), 2.59-2.51(2 \mathrm{H}, \mathrm{m})$, $2.48\left(2 \mathrm{H}, \mathrm{q}, J\right.$ 7.3), $1.19\left(3 \mathrm{H}, \mathrm{t}, J\right.$ 7.3); $\delta_{\mathrm{C}}\left(75 \mathrm{MHz}, \mathrm{CDCl}_{3}\right)$ 207.5, 173.6, 52.3, 43.2, $37.7,28.0,26.6,25.7,15.1 ; v_{\max }($ film $) / \mathrm{cm}^{-1} 2927,1737,1719,1438 ; \mathrm{m} / z$ (EI+) 204 (15\%, M+), 186, 143, 111; found 204.0814 [M] ${ }^{+} \mathrm{C}_{9} \mathrm{H}_{16} \mathrm{O}_{3} \mathrm{~S}$ requires 204.0815 .

\section{3-phenylsulfanyl-propionaldehyde, Table 3 entry 2 substrate}<smiles>O=CCC[SbH2]</smiles>

The general procedure for the aldehyde preparation was followed employing benzenethiol (12.3 g, $111 \mathrm{mmol})$, acrolein (5.0 g, $89 \mathrm{mmol})$, and $\mathrm{NaH}(18 \mathrm{mg}, 0.44$ mmol) to yield the tile compound as a colourless oil $(11.4 \mathrm{~g}, 66 \%) ; \delta_{\mathrm{H}}(300 \mathrm{MHz}$, $\left.\mathrm{CDCl}_{3}\right) 9.69(1 \mathrm{H}, \mathrm{s}), 7.32-7.05(5 \mathrm{H}, \mathrm{m}), 3.11(2 \mathrm{H}, \mathrm{t}, J 7.2), 2.70(2 \mathrm{H}, \mathrm{t}, J 7.2)$. Used directly in subsequent hydroacylation reactions.

\section{Methyl 4-oxo-6-phenylsulfanyl-hexanoate, Table 3 entry 2}<smiles>COC(=O)CCC(=O)CCc1ccccc1</smiles>

The general procedure for the hydroacylation reaction was followed employing precatalyst $\left[\mathrm{Rh}(\mathrm{nbd}) \mathrm{dppe}_{\mathrm{ClO}} \quad(10 \quad \mathrm{mg}, \quad 0.014 \quad \mathrm{mmol}), \quad 3-(\right.$ phenylsulfanyl)propionaldehyde (23 mg, $0.14 \mathrm{mmol})$ and methyacrylate $(65 \mu \mathrm{L}, 62 \mathrm{mg}, 0.70 \mathrm{mmol})$. Heated at $55{ }^{\circ} \mathrm{C}$ for 16 hours to give the title compound as a colourless oil (33 mg, 92 \%) Data in accordance with the literature ${ }^{2} \delta_{\mathrm{H}}\left(300 \mathrm{MHz}, \mathrm{CDCl}_{3}\right) 7.30-7.08(5 \mathrm{H}, \mathrm{m})$, $3.59(3 \mathrm{H}, \mathrm{s}), 3.59(2 \mathrm{H}, J$ 7.2), $2.71(2 \mathrm{H}, \mathrm{t}, J 7.2), 2.67-2.60(2 \mathrm{H}, \mathrm{m}) 2.55-2.48(2 \mathrm{H}$, $\mathrm{m}) ; \delta_{\mathrm{C}}\left(75 \mathrm{MHz}, \mathrm{CDCl}_{3}\right) 207.5,173.6,52.3,43.2,37.7,28.0,26.6,25.7,15.1 ; \mathrm{m} / \mathrm{z}$ $(\mathrm{EI}+) 252(100 \%, \mathrm{M}+), 221,165,137$; found $252.0816[\mathrm{M}]^{+} \mathrm{C}_{13} \mathrm{H}_{16} \mathrm{O}_{3} \mathrm{~S}$ requires 252.0816 .

\footnotetext{
${ }^{2}$ Grimm, E. L.; Zschwiesche, R.; Reissig, H.-U. J. Org. Chem. 1985, 50, 5543-5545.
} 


\section{3-Ethanesulfanyl-2-methyl-propionaldehyde, Table 3 entry 3 substrate}<smiles>CCSCC(C)C=O</smiles>

The general procedure for the aldehyde preparation was followed employing ethane thiol (1.9 g, $30.32 \mathrm{mmol})$, methacrolein (1.7 g, $24.25 \mathrm{mmol})$, and $\mathrm{NaH}$ (10 mg, 0.24 mmol) to yield the tile compound as a colourless oil $(2.40 \mathrm{~g}, 67 \%) ; \delta_{\mathrm{H}}(300 \mathrm{MHz}$, $\left.\mathrm{CDCl}_{3}\right) 9.62$ (1 H, d, J 1.5), 2.85-2.75 (1 H, m), 2.60-2.42 (2 H, m), $2.49(2 \mathrm{H}, \mathrm{q}, J$ 7.6), $1.20(3 \mathrm{H}, \mathrm{t}, J 7.6), 1.15(3 \mathrm{H}, \mathrm{d}, J 6.9)$. Used directly in subsequent hydroacylation reactions.

\section{6-Ethanesulfanyl-5-methyl-4-oxo-hexanoic acid methyl ester, Table 3 entry 3}<smiles>CCSCC(C)C(=O)CCC(=O)OC</smiles>

The general procedure for the hydroacylation reaction was followed employing precatalyst $[\mathrm{Rh}(\mathrm{nbd})(\mathrm{dppe})] \mathrm{ClO}_{4}(7 \mathrm{mg}, 0.01 \mathrm{mmol})$, acetone $(2.0 \mathrm{~mL})$, 3-ethanesulfanyl2-methyl-propionaldehyde ( $26 \mu \mathrm{L}, 0.2 \mathrm{mmol})$ and methyl acrylate $(70 \mu \mathrm{L}, 0.6 \mathrm{mmol})$. Heated at $55{ }^{\circ} \mathrm{C}$ for 16 hours. Flash chromatography (1:4, EtOAc:Petrol) yielded the title compound as a yellow oil (36 mg, $83 \%$ ); $v_{\max }$ (film) $/ \mathrm{cm}^{-1} 3045,2977,1742$, 1427, 1264, 753; $\delta_{\mathrm{H}}\left(300 \mathrm{MHz} ; \mathrm{CDCl}_{3}\right) 3.61(3 \mathrm{H}, \mathrm{s}), 2.86-2.64(4 \mathrm{H}, \mathrm{m}), 2.57-2.43$ $(5 \mathrm{H}, \mathrm{m}), 1.20-1.10(6 \mathrm{H}, \mathrm{m}) ; \delta_{\mathrm{C}}\left(75 \mathrm{MHz} ; \mathrm{CDCl}_{3}\right) 210.7,172.9,51.5,46.1,36.0,33.9$, 27.3, 26.4; m/z (EI) 218 (5 \%, M), 157 (90\%, M- $\left.\mathrm{H}_{2} \mathrm{CO}_{2} \mathrm{Me}\right), 125$ (100\%, EtSHOMe); found $[\mathrm{M}]^{+} 218.0971, \mathrm{C}_{10} \mathrm{H}_{18} \mathrm{O}_{3} \mathrm{~S}$ requires 218.0972 .

\section{3-Ethanesulfanyl-butyraldehyde, Table 3 entry 4 substrate}<smiles>CC([SiH3])CCC=O</smiles>

The general procedure for the aldehyde preparation was followed employing ethane thiol (5.5 g, $88.75 \mathrm{mmol}$ ), crotonaldehyde (5.0 g, $71.00 \mathrm{mmol}$ ), and $\mathrm{NaH} 15 \mathrm{mg}, 0.41$ mmol) to yield the tile compound as a colourless oil $(7.3 \mathrm{~g}, 70 \%) ; \delta_{\mathrm{H}}(300 \mathrm{MHz}$, $\left.\mathrm{CDCl}_{3}\right) 9.71(1 \mathrm{H}, \mathrm{t}, J 1.9), 3.23(1 \mathrm{H}, \mathrm{dq}, J 7.6,6.7), 2.67-2.50(3 \mathrm{H}, \mathrm{m}), 2.52(2 \mathrm{H}, \mathrm{q}$, 
$J$ 7.6), 1.29 (3 H, d, $J$ 6.7), $1.20(3 \mathrm{H}, \mathrm{d}, J$ 7.6). Used directly in subsequent hydroacylation reactions.

\section{6-Ethanesulfanyl-4-oxo-heptanoic acid methyl ester, Table 3 entry 4}<smiles>CCC(C)CC(=O)CCC(=O)OC</smiles>

The general procedure for the hydroacylation reaction was followed employing precatalyst $\left[\mathrm{Rh}(\mathrm{nbd})(\mathrm{dppe}) \mathrm{ClO}_{4} \quad(9 \mathrm{mg}, \quad 0.013 \mathrm{mmol})\right.$, acetone $(2.0 \mathrm{~mL}), 3-$ ethanesulfanyl-butyraldehyde $(33 \mathrm{mg}, 0.25 \mathrm{mmol})$ and methyl acrylate $(112 \mu \mathrm{L}, 0.75$ mmol). Heated at $55{ }^{\circ} \mathrm{C}$ for 16 hours. Flash chromatography (1:4, EtOAc:Petrol) yielded the title compound as a yellow oil (42 mg, $77 \%$ ); $v_{\max }$ (film) $/ \mathrm{cm}^{-1} 2950$, $1736,1652,1556,899,731 ; \delta_{\mathrm{H}}\left(300 \mathrm{MHz} ; \mathrm{CDCl}_{3}\right) 3.67(3 \mathrm{H}, \mathrm{s}), 3.34-3.22(1 \mathrm{H}, \mathrm{m})$, 2.79-2.71 (4H, m), 2.62-2.52 (4H, m), $1.28(3 \mathrm{H}, \mathrm{d}, J 6.8), 1.24(3 \mathrm{H}, \mathrm{t}, J 7.4) ; \delta_{\mathrm{C}}(75$ $\mathrm{MHz} ; \mathrm{CDCl}_{3}$ ) 206.7, 173.0, 51.7, 50.0, 37.8, 34.6, 27.5, 24.5, 21.5, 14.6; m/z (EI) 218 $(10 \%, \mathrm{M}), \quad 115(90 \% \text {, M-EtSMeCHCH})_{2} 89$ (100\% M- $\left.\mathrm{CH}_{2} \mathrm{COCH}_{2} \mathrm{CH}_{2} \mathrm{CO}_{2} \mathrm{Me}\right)$; found $[\mathrm{M}]^{+} 218.0968, \mathrm{C}_{10} \mathrm{H}_{18} \mathrm{O}_{3} \mathrm{~S}$ requires 218.0971.

\section{3-Ethanesulfanyl-3-phenyl-proionaldehyde, Table 3 entry 5 substrate}<smiles>O=CCC([Si])c1ccccc1</smiles>

The general procedure for the aldehyde preparation was followed employing ethane thiol (2.2 g, $34.0 \mathrm{mmol}$ ), cinnamaldehyde (3.0 g, $22.0 \mathrm{mmol})$, and $\mathrm{NaH}$ (8 mg, 0.22 mmol) to yield the tile compound as a colourless oil $(2.8 \mathrm{~g}, 66 \%) ; \delta_{\mathrm{H}}(300 \mathrm{MHz}$, $\left.\mathrm{CDCl}_{3}\right) 9.63(1 \mathrm{H}, \mathrm{t}, J 1.8), 7.40-71.5(5 \mathrm{H}, \mathrm{m}), 4.29(1 \mathrm{H}, \mathrm{t}, J 7.6), 2.88(2 \mathrm{H}, \mathrm{m})$, 2.36-2.18 (2 H, m), $1.09(3 \mathrm{H}, \mathrm{t}, J$ 7.6). Used directly in subsequent hydroacylation reactions.

\section{6-Ethanesulfanyl-4-oxo-6-phenyl-hexanoic acid methyl ester, Table 3 entry 5}<smiles>CCOC(CC(=O)CCC(=O)OC)c1ccccc1</smiles>

The general procedure for the hydroacylation reaction was followed employing precatalyst $[\mathrm{Rh}(\mathrm{nbd})(\mathrm{dppe})] \mathrm{ClO}_{4} \quad(5 \mathrm{mg}, \quad 0.0075 \mathrm{mmol})$, acetone $(2.0 \mathrm{~mL}), 3-$ ethanesulfanyl-3-phenyl-propionaldehyde (29 mg, $0.15 \mathrm{mmol})$ and methyl acrylate 
$(67 \mu \mathrm{L}, 0.45 \mathrm{mmol})$. Heated at $55{ }^{\circ} \mathrm{C}$ for 16 hours. Flash chromatography (1:4, EtOAc:Petrol) yielded the title compound as a yellow oil (37 mg $87 \%$ ); $v_{\max }$ (film) $/ \mathrm{cm}^{-1} 3060,1709,1658,1411,1252,903,718 ; \delta_{\mathrm{H}}\left(300 \mathrm{MHz} ; \mathrm{CDCl}_{3}\right) 7.29-7.12(5 \mathrm{H}$, m), $4.29(1 \mathrm{H}, \mathrm{t}, J 7.3), 3.56(3 \mathrm{H}, \mathrm{s}), 2.93(2 \mathrm{H}, \mathrm{d}, J 7.3), 2.71-2.43(4 \mathrm{H}, \mathrm{m}), 2.30-2.18$ $(2 \mathrm{H}, \mathrm{m}), 1.08(3 \mathrm{H}, \mathrm{t}, J 7.4) ; \delta_{\mathrm{C}}\left(75 \mathrm{MHz} ; \mathrm{CDCl}_{3}\right) 205.2,172.5,141.4,128.1(2 \mathrm{CH})$, 127.2 (2 CH), 126.8, 51.3, 48.8, 43.3, 37.5, 27.1, 24.9, 13.9; m/z (EI) 280 (10 \%, M), $115(100 \%, \mathrm{M}-\mathrm{EtSPhCHCH})$; found $[\mathrm{M}]^{+} 280.1126, \mathrm{C}_{15} \mathrm{H}_{20} \mathrm{O}_{3} \mathrm{~S}$ requires 280.1128 .

\section{3-Ethanesulfanyl-2-methyl-butyraldehyde, Table 3 entry 6 substrate}<smiles>CC(C)C(C)C=O</smiles>

The general procedure for the aldehyde preparation was followed employing ethane thiol (4.6 g, $74.3 \mathrm{mmol}$ ), 2-methylcrotonaldehyde (5.0 g, $59.43 \mathrm{mmol})$, and $\mathrm{NaH}$ (12 $\mathrm{mg}, 0.30 \mathrm{mmol}$ ) to yield the tile compound as a colourless oil as a 1:1 mixture of diastereomers $(4.5 \mathrm{~g}, 47 \%) ; \delta_{\mathrm{H}}\left(300 \mathrm{MHz}, \mathrm{CDCl}_{3}\right) ; 9.67(0.5 \mathrm{H}, \mathrm{d}, J 1.8), 9.61(0.5$ H, d, $J 1.8), 3.19-3.05$ (1 H, m), 2.67-2.40 (3 H, m), 1.29 (3 H, d $J 7.3) 1.19$ (3 H, t, $J$ 7.6), $1.10(1.5 \mathrm{H}, \mathrm{d}, J 7.2), 1.10(1.5 \mathrm{H}, \mathrm{d}, J 7.1)$. Used directly in subsequent hydroacylation reactions.

\section{Methyl 6-ethanesulfanyl-5-methyl-4-oxo-heptanoate, Table 3 entry 6}<smiles>CCC(C)C(C)C(=O)CCC(=O)OC</smiles>

The general procedure for the hydroacylation reaction was followed employing precatalyst $\quad[\mathrm{Rh}(\mathrm{nbd}) \mathrm{dppe}] \mathrm{ClO}_{4} \quad(10 \quad \mathrm{mg}, \quad 0.014 \quad \mathrm{mmol}), \quad 3$-(ethanesulfanyl)-2methybutryaldehyde $(20 \mathrm{mg}, 0.14 \mathrm{mmol})$ and methyacrylate $(65 \mu \mathrm{L}, 62 \mathrm{mg}, 0.70$ mmol). Heated at $55{ }^{\circ} \mathrm{C}$ for 20 hours to give the title compound as a colourless oil as a 1:1 mixture of diastereomers (25 mg, $77 \%) ; \delta_{\mathrm{H}}\left(300 \mathrm{MHz}, \mathrm{CDCl}_{3}\right)$ (Major Diastereomer) $3.61(3 \mathrm{H}, \mathrm{s}), 3.07-2.92(1 \mathrm{H}, \mathrm{m}), 2.80-2.72(2 \mathrm{H}, \mathrm{m}), 2.61-2.42(5 \mathrm{H}, \mathrm{m})$, 1.24-1.14 (6H, m), $1.07(3 \mathrm{H}, \mathrm{d}, J 6.7) ; \delta_{\mathrm{H}}\left(300 \mathrm{MHz}, \mathrm{CDCl}_{3}\right)$ (Minor Diasteromer) $3.61(3 \mathrm{H}, \mathrm{s}), 3.07-2.92(1 \mathrm{H}, \mathrm{m}), 2.80-2.72(2 \mathrm{H}, \mathrm{m}), 2.61-2.42(5 \mathrm{H}, \mathrm{m}), 1.24-1.1 .05$ $(9 \mathrm{H}, \mathrm{m}) ; \delta_{\mathrm{C}}\left(75 \mathrm{MHz}, \mathrm{CDCl}_{3}\right)$ 211.1, 210.8, 173.3, 173.3, 51.8, 51.7, 51.1, 42.3, 40.9, $37.1,36.8,31.9,28.3,27.62,27.59,25.3,24.8,20.3,18.1,14.8,14.73,14.68,13.0$; 
$v_{\max }\left(\right.$ film) $/ \mathrm{cm}^{-1} 2970,2903,1741,1714,1438 ; \mathrm{m} / z(\mathrm{EI}+) 232(5 \%, \mathrm{M}+), 171,138$, 115; found $232.1129[\mathrm{M}]^{+} \mathrm{C}_{11} \mathrm{H}_{20} \mathrm{O}_{3} \mathrm{~S}$ requires 232.1128.

(E)-6-Methanesulfanyl-4-oxo-hex-2-enoic acid ethyl ester, 4<smiles>CCOC(=O)/C=C/C(=O)CCS(C)(=O)=O</smiles>

The general procedure for the hydroacylation reaction was followed employing precatalyst $[\mathrm{Rh}(\mathrm{nbd})(\mathrm{dppe})] \mathrm{ClO}_{4}(10 \mathrm{mg}, 0.015 \mathrm{mmol})$, acetone $(2.0 \mathrm{~mL}), 3-$ methanesulfanyl propanal $(30 \mu \mathrm{L}, 0.3 \mathrm{mmol})$ and ethyl propiolate $(152 \mu \mathrm{L}, 1.5$ mmol). Heated at $55{ }^{\circ} \mathrm{C}$ for 1 hour. Flash chromatography $\left(\mathrm{CH}_{2} \mathrm{Cl}_{2}\right)$ yielded the title compound as a yellow oil (44 mg, $73 \%$ ); $v_{\max }(\mathrm{film}) / \mathrm{cm}^{-1}$ 2930, 1697 1629, 1245, $1030 ; \delta_{\mathrm{H}}\left(300 \mathrm{MHz} ; \mathrm{CDCl}_{3}\right) 7.00(1 \mathrm{H}, \mathrm{d}, J 16.0), 6.63(1 \mathrm{H}, \mathrm{d}, J 16.0), 4.20(2 \mathrm{H}, \mathrm{q}, J$ 7.1), 2.89 (2H, t, J 7.5), $2.73(2 \mathrm{H}, \mathrm{t}, J 7.5), 2.06(3 \mathrm{H}, \mathrm{s}), 1.26(3 \mathrm{H}, \mathrm{t}, J 7.1) ; \delta_{\mathrm{C}}(75$ $\mathrm{MHz} ; \mathrm{CDCl}_{3}$ ) 198.2, 165.8, 139.3, 131.7, 61.9, 41.7, 28.2, 16.3, 14.5; m/z (EI) 202 (15\%, M), 157 (40\%, M-OCH $\left.\mathrm{CH}_{3}\right), 61$ (100\%, M-MeSCH $\left.\mathrm{CH}_{2} \mathrm{COCHCHC}\right)$; found $[\mathrm{M}]^{+}$202.0658, $\mathrm{C}_{9} \mathrm{H}_{14} \mathrm{O}_{3} \mathrm{~S}$ required 202.0655.

\section{(E)-1-Methanesulfanyl-non-4-en-3-one, Table 5 entry 1}<smiles>CCCC/C=C/C(=O)CCS(C)(=O)=O</smiles>

The general procedure for the hydroacylation reaction was followed employing precatalyst $[\mathrm{Rh}(\mathrm{nbd})(\mathrm{dppe})] \mathrm{ClO}_{4}(10 \mathrm{mg}, 0.015 \mathrm{mmol})$, acetone $(2.0 \mathrm{~mL}), 3-$ methanesulfanyl propanal $(30 \mu \mathrm{L}, 0.3 \mathrm{mmol})$ and 1-hexyne $(160 \mu \mathrm{L}, 1.5 \mathrm{mmol})$. The resulting mixture was stirred and heated at $55{ }^{\circ} \mathrm{C}$ for 1 hour. Flash chromatography (1:4 EtOAc:Petrol) yielded the title compound as a yellow oil (54 mg, 98\%); $v_{\max }$ (film) $/ \mathrm{cm}^{-1} 3049,1704,1658,1421,1267,733 ; \delta_{\mathrm{H}}\left(300 \mathrm{MHz} ; \mathrm{CDCl}_{3}\right) 6.86(1 \mathrm{H}, \mathrm{dt}, J$ 15.9, 6.9), 6.10 (1H, dt, J 15.9, 1.4), 2.87-2.73 (4H, m), 2.26-2.18 (2H, m), $2.12(3 \mathrm{H}$, s), 1.48-1.30 (4H, m), $0.91(3 \mathrm{H}, \mathrm{t}, J 7.2) ; \delta_{\mathrm{C}}\left(75 \mathrm{MHz} ; \mathrm{CDCl}_{3}\right)$ 198.4, 148.1, 129.9, 39.6, 32.1, 30.0, 28.2, 22.1, 15.7, 13.7; m/z (EI) 186 (20\%, M), 129 (40\%, M$\left.\mathrm{C}_{3} \mathrm{H}_{6} \mathrm{CH}_{3}\right) 55\left(100 \%, \mathrm{M}-\mathrm{MeSCH}_{2} \mathrm{CH}_{2} \mathrm{COCHCH}_{3}\right)$; found $[\mathrm{M}]^{+} 186.1073, \mathrm{C}_{10} \mathrm{H}_{18} \mathrm{O}$ requires 186.1073 . 
(E)-8-Chloro-2-methanesulfanyl-oct-4-en-3-one, Table 5 entry 3<smiles>CSCCC(=O)C=CCCCCl</smiles>

The general procedure for the hydroacylation reaction was followed employing precatalyst $[\mathrm{Rh}(\mathrm{nbd})(\mathrm{dppe})] \mathrm{ClO}_{4}(10 \mathrm{mg}, 0.015 \mathrm{mmol})$, acetone $(2.0 \mathrm{~mL}), 3-$ methanesulfanyl propanal $(30 \mu \mathrm{L}, 0.3 \mathrm{mmol})$ and 5-chloro-1-pentyne $(158 \mu \mathrm{L}, 1.5$ mmol). Heated at $55^{\circ} \mathrm{C}$ for 1 hour. Flash chromatography (1:4, EtOAc:Petrol) yielded the title compound as a yellow oil (53 mg, $85 \%) ; v_{\max }\left(\right.$ film) $/ \mathrm{cm}^{-1} 3049,1632,1416$, 1252,$733 ; \delta_{\mathrm{H}}\left(300 \mathrm{MHz} ; \mathrm{CDCl}_{3}\right) 6.83(1 \mathrm{H}, \mathrm{dt}, J 15.9,6.9), 6.17(1 \mathrm{H}, \mathrm{dt}, J 15.9,1.5)$, 3.56 (2H, t $J 6.4), 2.88-2.74(4 \mathrm{H}, \mathrm{m}), 2.45-2.37(2 \mathrm{H}, \mathrm{m}), 2.12(3 \mathrm{H}, \mathrm{s}), 1.99-1.91(2 \mathrm{H}$, $\mathrm{m}) ; \delta_{\mathrm{C}}\left(75 \mathrm{MHz} ; \mathrm{CDCl}_{3}\right)$ 197.7, 145.1, 130.3, 43.5, 39.6, 30.2, 29.0, 27.8, 15.4; $v_{\max }$ (film) $/ \mathrm{cm}^{-1} 1669$ (C=O), 735 (C-Cl); m/z (EI) 206 (20\%, M), 159 (60\% M-OCO) $131\left(100 \%, \mathrm{M}-\mathrm{CH}_{4} \mathrm{CO}_{2} \mathrm{Me}\right)$; found $[\mathrm{M}]^{+}$206.0527, $\mathrm{C}_{9} \mathrm{H}_{15} \mathrm{ClOS}$ requires 206.0527.

\section{(E)-8-Hydroxy-1-methanesulfanyl-oct-4-en-3-one, Table 5 entry 5}<smiles>CSCCC(=O)C=CCCCO</smiles>

The general procedure for the hydroacylation reaction was followed employing precatalyst $[\mathrm{Rh}(\mathrm{nbd})(\mathrm{dppe})] \mathrm{ClO}_{4}(10 \mathrm{mg}, \quad 0.015 \mathrm{mmol})$, acetone $(2.0 \mathrm{~mL}), 3-$ methanesulfanyl propanal $(30 \mu \mathrm{L}, 0.3 \mathrm{mmol})$ and 4-pentyn-1-ol $(56 \mu \mathrm{L}, 0.6 \mathrm{mmol})$. Heated at $55{ }^{\circ} \mathrm{C}$ for 1 hour. Flash chromatography (1:1, EtOAc:Petrol) yielded the title compound as a colourless oil (54 mg, $95 \%$ ); $v_{\max }(\mathrm{film}) / \mathrm{cm}^{-1} 3415,2921,1669$, $1618,1399,1051 ; \delta_{\mathrm{H}}\left(300 \mathrm{MHz} ; \mathrm{CDCl}_{3}\right) 6.86(1 \mathrm{H}, \mathrm{dt}, J 15.9,6.9), 6.12(1 \mathrm{H}, \mathrm{dt}, J$ $15.9,1.5), 3.65(2 \mathrm{H}, \mathrm{t}, J 6.3), 2.86-2.81(2 \mathrm{H}, \mathrm{m}), 2.76-2.71(2 \mathrm{H}, \mathrm{m}), 2.35-2.28(2 \mathrm{H}$, m), $2.10(3 \mathrm{H}, \mathrm{s}), 1.86\left(1 \mathrm{H}\right.$, br. s), 1.76-1.67 $(2 \mathrm{H}, \mathrm{m}) ; \delta_{\mathrm{C}}\left(75 \mathrm{MHz} ; \mathrm{CDCl}_{3}\right)$ 197.9, $146.7,129.6,61.2,39.1,30.2,28.2,27.6,15.1 ; \mathrm{m} / z$ (EI) 188 (3\%, M), 140 (80\%, M$\mathrm{MeSH}) 71\left(100 \% \mathrm{M}-\mathrm{MeSCH}_{2} \mathrm{CH}_{2} \mathrm{CO}\right)$; found $[\mathrm{M}]^{+}$218.0968, $\mathrm{C}_{10} \mathrm{H}_{18} \mathrm{O}_{3} \mathrm{~S}$ requires 218.0971 . 
(E)-8-(tert-Butyl-dimethyl-silyloxy-1-methanesulfanyl-oct-4-en-3-one, Table 5 entry 6<smiles>CSCCC(=O)C=CCCC[Os+2]</smiles>

The general procedure for the hydroacylation reaction was followed employing precatalyst $\left[\mathrm{Rh}(\mathrm{nbd})(\mathrm{dppe}) \mathrm{ClO}_{4}(10 \mathrm{mg}, \quad 0.015 \mathrm{mmol})\right.$, acetone $(2.0 \mathrm{~mL}), 3-$ methanesulfanyl propanal $(30 \mu \mathrm{L}, 0.3 \mathrm{mmol})$ and $t$-butyl-dimethyl-pent-4-ynyloxysilane (136 mg, $0.6 \mathrm{mmol})$. Heated at $55{ }^{\circ} \mathrm{C}$ for 1 hour. Flash chromatography (1:3, EtOAc:Petrol) yielded the title compound as a pale yellow oil $(81 \mathrm{mg}, 89 \%)$; $v_{\max }$ (film) $/ \mathrm{cm}^{-1} 2921,1653,1345,667 ; \delta_{\mathrm{H}}\left(300 \mathrm{MHz} ; \mathrm{CDCl}_{3}\right) 6.86(1 \mathrm{H}, \mathrm{dt}, J 15.9,6.9)$, 6.09 (1H, dt, $J 15.9,1.5), 3.60(2 \mathrm{H}, \mathrm{t}, J 6.1), 2.85-2.71(4 \mathrm{H}, \mathrm{m}), 2.32-2.24(2 \mathrm{H}, \mathrm{m})$, $2.09(3 \mathrm{H}, \mathrm{s}), 1.70-1.61(2 \mathrm{H}, \mathrm{m}), 0.86(9 \mathrm{H}, \mathrm{s}), 0.02(6 \mathrm{H}, \mathrm{s}) ; \delta_{\mathrm{C}}\left(75 \mathrm{MHz} ; \mathrm{CDCl}_{3}\right)$ 197.8, 147.1, 129.5, 61.5, 39.1, 30.4, 28.4, 27.6, $25.2\left(3 \mathrm{CH}_{3}\right), 17.6,15.2,-5.9$ (2 $\left.\mathrm{CH}_{3}\right) ; m / z(\mathrm{CI}+) 303(100 \%, \mathrm{M}+\mathrm{H}), 255$ (40\%, M-MeS); found [M] 303.1809, $\mathrm{C}_{15} \mathrm{H}_{30} \mathrm{O}_{2} \mathrm{SSi}$ requires 303.1809.

\section{(E)-1-Methanesulfanyl-8-(tetrahydro-pyran-2-yloxy)-oct-4-en-3-one, Table 5} entry 7<smiles>CSCCC(=O)C=CCCC[OH+]</smiles>

The general procedure for the hydroacylation reaction was followed employing precatalyst $[\mathrm{Rh}(\mathrm{nbd})(\mathrm{dppe})] \mathrm{ClO}_{4}(10 \mathrm{mg}, \quad 0.015 \mathrm{mmol})$, acetone $(2.0 \mathrm{~mL}), 3-$ methanesulfanyl propanal $(30 \mu \mathrm{L}, 0.3 \mathrm{mmol})$ and 2-pent-4-ynyloxy-tetrahydro-pyran (118 mg, $0.6 \mathrm{mmol})$. Heated at $55{ }^{\circ} \mathrm{C}$ for 1 hour. Flash chromatography (1:3, EtOAc:Petrol) yielded the title compound as a pale yellow oil (76 mg, $93 \%)$; $v_{\max }$ (film) $/ \mathrm{cm}^{-1} 2961,2865,1686,1276,911 ; \delta_{\mathrm{H}}\left(300 \mathrm{MHz} ; \mathrm{CDCl}_{3}\right) 6.88(1 \mathrm{H}, \mathrm{dt}, J$ 15.9, 6.9), $6.11(1 \mathrm{H}, \mathrm{dt}, J 15.9,1.5), 4.55(1 \mathrm{H}, \mathrm{t}, J 3.0), 3.87-3.71(2 \mathrm{H}, \mathrm{m}), 3.52-3.36(2 \mathrm{H}$, m), 2.87-2.71 (4H, m), 2.36-2.29 (2H, m), $2.10(3 \mathrm{H}, \mathrm{s}), 1.83-1.69(4 \mathrm{H}, \mathrm{m}), 1.58-1.47$ $(4 \mathrm{H}, \mathrm{m}) ; \delta_{\mathrm{C}}\left(75 \mathrm{MHz} ; \mathrm{CDCl}_{3}\right)$ 198.3, 147.3, 130.0, 98.7, 66.3, 62.2, 39.5, 30.4, 29.2, 28.1, 27.9, 25.2, 19.4, 15.6; m/z (EI) 272 (5\%, M), 225 (40\%, M-MeS), 172 (80\%, MOTHP), 169 (100\% M-MeSCH${ }_{2} \mathrm{CH}_{2} \mathrm{CO}$ ); found [M] ${ }^{+} 272.1442, \mathrm{C}_{14} \mathrm{H}_{24} \mathrm{O}_{3} \mathrm{~S}$ requires 272.1446 . 


\section{(E)-10-Methanesulfanyl-dec-6-ene-2,8-dione Table 5 entry 8}<smiles>CC(=O)CCCC=CC(=O)CCS(C)(=O)=O</smiles>

The general procedure for the hydroacylation reaction was followed employing precatalyst $[\mathrm{Rh}(\mathrm{nbd})(\mathrm{dppe})] \mathrm{ClO}_{4}(10 \mathrm{mg}, \quad 0.015 \mathrm{mmol})$, acetone $(2.0 \mathrm{~mL}), 3-$ methanesulfanyl propanal $(30 \mu \mathrm{L}, 0.3 \mathrm{mmol})$ and hept-6-yn-2-one (66 mg, $0.6 \mathrm{mmol})$. Heated at $55{ }^{\circ} \mathrm{C}$ for 1 hour. Flash chromatography (1:4, EtOAc:Petrol) yielded the title compound as a yellow oil (44 mg, 69\%); $v_{\max }\left(\right.$ film) $/ \mathrm{cm}^{-1} 3049,2978,2295$, 1704, 1673, 1617, 1411, 1252, 903, 744; $\delta_{\mathrm{H}}\left(300 \mathrm{MHz} ; \mathrm{CDCl}_{3}\right) 6.82(1 \mathrm{H}, \mathrm{dt}, J 15.9$, 6.6), $6.11(1 \mathrm{H}, \mathrm{dt}, J 15.9,1.5), 2.88-2.74(4 \mathrm{H}, \mathrm{m}), 2.47$ (2H, t, J 7.2), 2.28-2.20 (2H, m), $2.14(3 \mathrm{H}, \mathrm{s}), 2.12(3 \mathrm{H}, \mathrm{s}), 1.81-1.71(2 \mathrm{H}, \mathrm{m}) ; \delta_{\mathrm{C}}\left(75 \mathrm{MHz} ; \mathrm{CDCl}_{3}\right) 207.8,198.1$, 146.6, 130.3, 42.3, 39.6, 31.4, 29.8, 28.0, 21.6, 15.6; m/z (EI+) 214 (5\%, M+), 73 (90\%, M-COCHCH $\left.\left(\mathrm{CH}_{2}\right)_{3} \mathrm{COCH}_{3}\right), 59\left(100 \%, \quad \mathrm{M}-\mathrm{CH}_{2} \mathrm{COCHCH}\left(\mathrm{CH}_{2}\right)_{3} \mathrm{COCH}_{3}\right)$; found 214.1022 [M] $]^{+} \mathrm{C}_{11} \mathrm{H}_{18} \mathrm{O}_{2} \mathrm{~S}$ requires 214.1022.

\section{(E)-1-Methanesulfanyl-dec-4-en-9-yn-3-one, Table 5 entry 9<smiles>C#CCCCC=CC(=O)CCSC</smiles>

The general procedure for the hydroacylation reaction was followed employing precatalyst $[\mathrm{Rh}(\mathrm{nbd})(\mathrm{dppe})] \mathrm{ClO}_{4}(10 \mathrm{mg}, \quad 0.015 \mathrm{mmol})$, acetone $(2.0 \mathrm{~mL}), 3-$ methanesulfanyl propanal $(30 \mu \mathrm{L}, 0.3 \mathrm{mmol})$ and 1,6-heptadiyne $(104 \mu \mathrm{L}, 0.9 \mathrm{mmol})$. Heated at $55{ }^{\circ} \mathrm{C}$ for 1 hour. Flash chromatography (1:4, EtOAc:Petrol) yielded the title compound as a pale yellow oil (44 mg, $74 \%) ; v_{\max }\left(\right.$ film) $/ \mathrm{cm}^{-1} 2976,1652,916$, $731 ; \delta_{\mathrm{H}}\left(300 \mathrm{MHz} ; \mathrm{CDCl}_{3} 6.85(1 \mathrm{H}, \mathrm{dt}, J 15.9,6.9), 6.15(1 \mathrm{H}, \mathrm{dt}, J 15.9,1.5), 2.88-\right.$ $2.74(4 \mathrm{H}, \mathrm{m}), 2.40-2.32(2 \mathrm{H}, \mathrm{m}), 2.24(2 \mathrm{H}, \mathrm{td}, J 7.0,2.6), 2.12(3 \mathrm{H}, \mathrm{s}), 1.98(1 \mathrm{H}, \mathrm{t}, J$ 2.6), 1.76-1.66 (2H, m); $\delta_{\mathrm{C}}\left(75 \mathrm{MHz} ; \mathrm{CDCl}_{3}\right)$ 198.3, 146.5, 83.4, 69.1, 39.9, 31.2, 28.3, 26.7, 17.9, 15.8; m/z (ES+) 196 (5\%, M), 181 (40\%, M-CH $\mathrm{CH}_{3}, 81$ (100\%, M$\left.\mathrm{CHC}\left(\mathrm{CH}_{2}\right)_{4}\right), 61\left(90 \%, \mathrm{M}-\mathrm{CHC}\left(\mathrm{CH}_{2}\right)_{3} \mathrm{CHCHCOCH}_{2}\right)$; found $[\mathrm{M}+\mathrm{H}]^{+}$197.0993, $\mathrm{C}_{11} \mathrm{H}_{17} \mathrm{OS}$ requires 197.0995 . 
(4E, 9E)-1,13-bis(methaneulfonyl)trideca-4,9-diene-3,11-dione, Table 5 entry 10<smiles>CSCCC(=O)C=CCCCCC=CC(=O)CCS(C)(C)C</smiles>

The general procedure for the hydroacylation reaction was followed employing precatalyst $[\mathrm{Rh}(\mathrm{nbd})(\mathrm{dppe})] \mathrm{ClO}_{4}(10 \mathrm{mg}, 0.015 \mathrm{mmol})$, acetone $(2.0 \mathrm{~mL}), 3-$ methanesulfanyl propanal $(30 \mu \mathrm{L}, 0.3 \mathrm{mmol})$ and 1,6-heptadiyne $(17 \mu \mathrm{L}, 0.15 \mathrm{mmol})$. Heated at $55{ }^{\circ} \mathrm{C}$ for 1 hour. Flash chromatography (1:4, EtOAc:Petrol) yielded the title compound as a pale yellow oil (27 mg, $60 \%) ; v_{\max }\left(\right.$ film) $/ \mathrm{cm}^{-1} 2980,1700,1650$, $900 ; \delta_{\mathrm{H}}\left(300 \mathrm{MHz} ; \mathrm{CDCl}_{3} 6.8(\mathrm{H}, \mathrm{dt}, J 15.8,6.98), 6.06(2 \mathrm{H}, \mathrm{dt}, J 15.8,1.5), 2.81-\right.$ $2.76(4 \mathrm{H}, \mathrm{m}), 2.76-2.73(4 \mathrm{H}, \mathrm{m}) 2.24-2.16(4 \mathrm{H}, \mathrm{m}), 2.06(6 \mathrm{H}, \mathrm{s}), 1.65-1.58(2 \mathrm{H}, \mathrm{m})$; $\delta_{\mathrm{C}}\left(75 \mathrm{MHz} ; \mathrm{CDCl}_{3}\right) 198.7(2 \mathrm{C}), 146.9(2 \mathrm{CH}), 131.0(2 \mathrm{CH}), 40.4\left(2 \mathrm{CH}_{2}\right), 32.2(2$ $\left.\mathrm{CH}_{2}\right), 28.7\left(2 \mathrm{CH}_{2}\right), 26.8\left(2 \mathrm{CH}_{2}\right)$ 16.3; $\mathrm{m} / z\left(\mathrm{CI}+, \mathrm{NH}_{3}\right) 318\left(7 \%, \mathrm{M}+\mathrm{NH}_{4}\right), 272$ $\left(100 \%, \quad \mathrm{M}-\mathrm{SCH}_{3}\right), 224\left(30 \%, \quad \mathrm{M}-\mathrm{CH}_{3} \mathrm{SCH}_{2} \mathrm{CH}_{3}\right)$; found $[\mathrm{M}+\mathrm{H}]^{+}$318.1557, $\mathrm{C}_{15} \mathrm{H}_{28} \mathrm{O}_{2} \mathrm{~N}_{1} \mathrm{~S}_{2}$ requires 318.1556 .

\section{(E)-5-Methanesulfanyl-1-trimethylsilanyl-pent-1-en-3-one, Table 5 entry 11}<smiles>CSC=CC(=O)CCSC</smiles>

The general procedure for the hydroacylation reaction was followed employing precatalyst $[\mathrm{Rh}(\mathrm{nbd})(\mathrm{dppe})] \mathrm{ClO}_{4}(10 \mathrm{mg}, \quad 0.015 \mathrm{mmol})$, acetone $(2.0 \mathrm{~mL}), 3-$ methanesulfanyl propanal $(30 \mu \mathrm{L}, 0.3 \mathrm{mmol})$ and (trimethylsilyl)acetylene $(85 \mu \mathrm{L}$, $0.6 \mathrm{mmol})$. Heated at $55{ }^{\circ} \mathrm{C}$ for 1 hour. Flash chromatography (1:4, EtOAc:Petrol) yielded the title compound as a pale yellow oil (53 mg, 89\%); $v_{\max }$ (film) $/ \mathrm{cm}^{-1} 2957$, $1678,1432,1250,995,836 ; \delta_{\mathrm{H}}\left(300 \mathrm{MHz}, \mathrm{CDCl}_{3}\right) 7.06(1 \mathrm{H}, \mathrm{d}, J 19.2), 6.45(1 \mathrm{H}, \mathrm{d}, J$ 19.2), 2.92-2.87 (2H, m), 2.77-2.72 (2H, m), $2.10(3 \mathrm{H}, \mathrm{s}), 0.12(9 \mathrm{H}, \mathrm{s}) ; \delta_{\mathrm{C}}(75 \mathrm{MHz}$, $\left.\mathrm{CDCl}_{3}\right)$ 198.4, 147.4, 141.8, 39.1, 28.1, 15.8, -1.9 (3C); m/z (ES+) 202 (10\%, M+), 139 (40\%, M-MeSCH${ }_{2} \mathrm{CH}_{2}$ ), 73 (80\%, M- $\left.\mathrm{MeSCH}_{2} \mathrm{CH}_{2} \mathrm{COCHCH}\right)$; found 203.0918 $[\mathrm{M}+\mathrm{H}]^{+} \mathrm{C}_{9} \mathrm{H}_{19} \mathrm{OSSi}$ requires 203.0920. 
(E)-1-Methanesulfanyl-4-methyl-hex-4-en-3-one, Table 5 entry 12<smiles>CC=C(C)C(=O)CCS(C)(=O)=O</smiles>

The general procedure for the hydroacylation reaction was followed employing precatalyst $[\mathrm{Rh}(\mathrm{nbd})(\mathrm{dppe})] \mathrm{ClO}_{4}(10 \mathrm{mg}, 0.015 \mathrm{mmol})$, acetone $(2.0 \mathrm{~mL}), 3-$ methanesulfanyl propanal $(30 \mu \mathrm{L}, 0.3 \mathrm{mmol})$ and 2-butyne $(71 \mu \mathrm{L}, 0.9 \mathrm{mmol})$. Heated at $55{ }^{\circ} \mathrm{C}$ for 16 hours. Flash chromatography (1:4, EtOAc:Petrol) yielded the title compound as a pale yellow oil (36 mg, $76 \%$ ); $v_{\max }\left(\right.$ film) $/ \mathrm{cm}^{-1} 2916,1663,1421$, 1242, 908, 723; $\delta_{\mathrm{H}}\left(300 \mathrm{MHz} ; \mathrm{CDCl}_{3}\right) 6.70(1 \mathrm{H}, \mathrm{qd}, J 6.9,1.1), 2.89(2 \mathrm{H}, \mathrm{t}, J$ 7.4), $2.70\left(2 \mathrm{H}, \mathrm{t}, J\right.$ 7.4), $2.06(3 \mathrm{H}, \mathrm{s}), 1.81(3 \mathrm{H}, \mathrm{d}, J 6.9), 1.72(3 \mathrm{H}, \mathrm{d}, J 1.1) ; \delta_{\mathrm{C}}(75 \mathrm{MHz}$; $\left.\mathrm{CDCl}_{3}\right)$ 200.1, 138.6, 138.2, 37.5, 29.5, 16.3, 15.3, 11.4; $\mathrm{m} / z$ (EI) 158 (10\%, M), 110 (100\%, M-CH 3 SH), $83\left(80 \%, \mathrm{M}-\mathrm{MeSCH}_{2} \mathrm{CH}_{2}\right), 61\left(40 \%, \mathrm{M}-\mathrm{CH}_{3} \mathrm{CHC}\left(\mathrm{CH}_{3}\right) \mathrm{COCH}_{2}\right)$; found [M] 158.0760, $\mathrm{C}_{8} \mathrm{H}_{14} \mathrm{OS}$ requires 158.0760.

\section{(E)-1-Methanesulfanyl-4-phenyl-hex-4-en-3-one, Table 5 entry 13}<smiles>CCCC(=O)C(=Cc1ccccc1)C(C)C</smiles>

The general procedure for the hydroacylation reaction was followed employing precatalyst $[\mathrm{Rh}(\mathrm{nbd})(\mathrm{dppe})] \mathrm{ClO}_{4} \quad(5 \mathrm{mg}, 0.0075 \mathrm{mmol})$, acetone $(1.5 \mathrm{~mL}), 3-$ methanesulfanyl propanal $(15 \mu \mathrm{L}, 0.15 \mathrm{mmol})$ and 1-phenyl-1-propyne $(56 \mu \mathrm{L}, 0.45$ mmol). Heated at $55{ }^{\circ} \mathrm{C}$ for 16 hours. Flash chromatography (1:4, EtOAc:Petrol) yielded the title compound as a pale yellow oil (22 mg, 67\%); $v_{\max }\left(\right.$ film) $/ \mathrm{cm}^{-1} 2916$, 2346, 1668, 1647, 1432, 1267, 1047, 728; $\delta_{\mathrm{H}}\left(300 \mathrm{MHz} ; \mathrm{CDCl}_{3}\right) 7.54$ (1H, d, J 1.4), 7.43-7.35 (5H, m), 3.14 (2H, t, J 7.6), 2.85 (2H, t, $J 7.6), 2.17(3 \mathrm{H}, \mathrm{s}), 2.07$ (3H, d, $J$ 1.4); $\delta_{\mathrm{C}}\left(75 \mathrm{MHz} ; \mathrm{CDCl}_{3}\right) 200.2,138.8,137.0,135.5,129.5(2 \mathrm{CH}), 128.4,128.3$ (2CH), 37.4, 28.9, 15.7, 12.9; m/z (ES+) $220(20 \%, \mathrm{M}+) 172(10 \%, \mathrm{M}-\mathrm{MeSH})$; found $221.0996[\mathrm{M}+\mathrm{H}]^{+} \mathrm{C}_{9} \mathrm{H}_{16} \mathrm{O}_{3} \mathrm{~S}$ requires 221.0995. 
(Z)-2-Benzylidene-5-methanesulfanyl-1-phenyl-pentane-1,3-dione, Table 5 entry 14<smiles>CCCC(=O)/C(=C\C(=O)c1ccccc1)c1ccccc1</smiles>

The general procedure for the hydroacylation reaction was followed employing precatalyst $[\mathrm{Rh}(\mathrm{nbd})(\mathrm{dppe})] \mathrm{ClO}_{4}(5 \mathrm{mg}, 0.00075 \mathrm{mmol})$, acetone $(1.5 \mathrm{~mL}), 3-$ methanesulfanyl propanal $(15 \mu \mathrm{L}, 0.15 \mathrm{mmol})$ and diphenylpropynone $(93 \mu \mathrm{L}, 0.45$ mmol). Heated at $55{ }^{\circ} \mathrm{C}$ for 16 hours. Flash chromatography (1:4, EtOAc:Petrol) yielded the title compound as a pale yellow oil (31 mg, $66 \%$ ); $v_{\max }\left(\right.$ film) $/ \mathrm{cm}^{-1} 2911$, 1673, 1647, 1581, 1221, 913; $\delta_{\mathrm{H}}\left(300 \mathrm{MHz} ; \mathrm{CDCl}_{3}\right) 7.88-7.84(2 \mathrm{H}, \mathrm{m}), 7.77(1 \mathrm{H}, \mathrm{s})$, 7.50-7.10 (8H, m), $2.92(2 \mathrm{H}, \mathrm{t}, J 7.0), 2.73(2 \mathrm{H}, \mathrm{t}, J 7.0), 2.01(3 \mathrm{H}, \mathrm{s}) ; \delta_{\mathrm{C}}(75 \mathrm{MHz}$; $\left.\mathrm{CDCl}_{3}\right) 198.3,196.5,141.9,139.3,136.3,134.6,133.2,131.0,130.8(2 \mathrm{CH}), 129.7$ (2CH), $129.4(2 \mathrm{CH}), 129.2(2 \mathrm{CH}), 40.0,28.7,16.3 ; \mathrm{m} / z(\mathrm{ES}+) 310(100 \%, \mathrm{M}+), 263$ $(60 \%, \mathrm{M}-\mathrm{MeS})$; found $311.1098[\mathrm{M}+\mathrm{H}]^{+} \mathrm{C}_{19} \mathrm{H}_{19} \mathrm{O}_{2} \mathrm{~S}$ requires 311.1100 .

(E)-6-Hydroxy-4-(hydroxymethyl)-1-methanesulfanyl-hex-4-en-3-one, Table 5 entry 15<smiles>CSCCC(=O)C(=CCO)CO</smiles>

The general procedure for the hydroacylation reaction was followed employing precatalyst $[\mathrm{Rh}(\mathrm{nbd})(\mathrm{dppe})] \mathrm{ClO}_{4}(10 \mathrm{mg}, 0.015 \mathrm{mmol})$, acetone $(2.0 \mathrm{~mL}), 3-$ methanesulfanyl propanal $(30 \mu \mathrm{L}, 0.3 \mathrm{mmol})$ and 2-butyne-1,4-diol (180 mg, 0.9 mmol). Heated at $55{ }^{\circ} \mathrm{C}$ for 16 hours. Flash chromatography (1:4, EtOAc:Petrol) yielded the title compound as a yellow oil (48 mg, $85 \%$ ); $v_{\max }\left(\right.$ film) $/ \mathrm{cm}^{-1} 2921$, 2860, 1653, 1354, 1043; $\delta_{\mathrm{H}}\left(300 \mathrm{MHz} ; \mathrm{CDCl}_{3}\right) 6.66(1 \mathrm{H}, \mathrm{tt}, J 3.7,1.3), 4.50(2 \mathrm{H}, \mathrm{dt}, J$ 3.7, 2.0), 4.38 (2H, td, J 2.0, 1.3), 2.87 (2H, t, J 7.1), 2.69 (2H, t, J 7.1), 2.05 (3H, s); $\delta_{\mathrm{C}}\left(75 \mathrm{MHz} ; \mathrm{CDCl}_{3}\right) 198.2,142.0,141.4,61.1,59.9,37.9,29.0,24.0,16.3 ; \mathrm{m} / z(\mathrm{ES}+)$ 190 (10\%, M+), 143 (60\%, M-MeS), 115 (100\%, M-MeSCH $\left.\mathrm{CH}_{2}\right), 87$ (40\%, M$\mathrm{MeSCH}_{2} \mathrm{CH}_{2} \mathrm{CO}$ ); found $191.5876[\mathrm{M}+\mathrm{H}]^{+} \mathrm{C}_{8} \mathrm{H}_{15} \mathrm{O}_{3} \mathrm{~S}$ requires 191.5874 . 
2-(-3-Methanesulfanyl-propionyl)-but-2-enedioic acid dimethyl ester Table 5 entry 17<smiles>COC(=O)C=C(C(=O)CCSC)C(=O)OC</smiles>

The general procedure for the hydroacylation reaction was followed employing precatalyst $\left[\mathrm{Rh}(\mathrm{nbd})(\mathrm{dppe}) \mathrm{ClO}_{4}(10 \mathrm{mg}, \quad 0.015 \mathrm{mmol})\right.$, acetone $(2.0 \mathrm{~mL}), 3-$ methanesulfanyl propanal $(30 \mu \mathrm{L}, 0.3 \mathrm{mmol})$ and dimethyl acetylenedicarboxylate $(158 \mu \mathrm{L}, 1.5 \mathrm{mmol})$. Heated at $55{ }^{\circ} \mathrm{C}$ for 16 hours. Flash chromatography (DCM) yielded the title compound as a yellow oil (55 mg, $75 \% 2: 1 \mathrm{Z:E}) ; v_{\max }(\mathrm{film}) / \mathrm{cm}^{-1}$ 3427, 3011, 2966, 2489, 1731,1728, 1455, 1248, 1051; $\delta_{\mathrm{H}}\left(300 \mathrm{MHz} ; \mathrm{CDCl}_{3}\right.$ ) (major Z) $6.80(1 \mathrm{H}, \mathrm{s}), 3.83(3 \mathrm{H}, \mathrm{s}), 3.78(3 \mathrm{H}, \mathrm{s}), 3.03-2.96(2 \mathrm{H}, \mathrm{m}), 2.90-2.84(2 \mathrm{H}, \mathrm{m}), 2.14$ $(3 \mathrm{H}, \mathrm{s})$; (minor E) $6.72(1 \mathrm{H}, \mathrm{s}), 3.91(3 \mathrm{H}, \mathrm{s}), 3.81(3 \mathrm{H}, \mathrm{s}), 3.03-2.96(2 \mathrm{H}, \mathrm{m}), 2.77$ $(2 \mathrm{H}, \mathrm{t}, J 7.3), 2.12(3 \mathrm{H}, \mathrm{s}) ; \delta_{\mathrm{C}}\left(75 \mathrm{MHz} ; \mathrm{CDCl}_{3}\right)(E$ and $Z) 199.6,193.1,164.7,163.5$, 163.4, 162.0, 145.7, 143.0, 127.7, 127.2, 52.2, 52.0, 51.8, 51.7, 41.8, 38.6, 26.6, 26.3, 14.9, 14.7; m/z (EI) $246(10 \%, \mathrm{M}), 167\left(100 \%, \mathrm{M}-\mathrm{MeSO}_{2}\right)$; found $[\mathrm{M}]^{+} 246.0556$, $\mathrm{C}_{10} \mathrm{H}_{14} \mathrm{O}_{5} \mathrm{~S}$ required 246.0555 .

\section{5-(3-Methanesulfanyl-propionyl)-hex-5-enenitrile, 6}<smiles>C=C(CCCC#N)C(=O)CCS(C)(=O)=O</smiles>

The general procedure for the hydroacylation reaction was followed employing precatalyst $[\mathrm{Rh}(\mathrm{nbd})(\mathrm{dppe})] \mathrm{ClO}_{4}(10 \mathrm{mg}, \quad 0.015 \mathrm{mmol})$, acetone $(2.0 \mathrm{~mL}), 3-$ methanesulfanyl propanal $(30 \mu \mathrm{L}, 0.3 \mathrm{mmol})$ and 5-hexynenitrile $(157 \mu \mathrm{L}, 1.5 \mathrm{mmol})$. Heated at $55{ }^{\circ} \mathrm{C}$ for 1 hour. Flash chromatography (1:1, EtOAc:Petrol) yielded the title compound as a yellow oil (41 mg, $68 \%$ ); $v_{\max }\left(\right.$ film) $/ \mathrm{cm}^{-1}$ 2967, 2337, 1646, 1551, 1123, 750; $\delta_{\mathrm{H}}\left(300 \mathrm{MHz} ; \mathrm{CDCl}_{3}\right) 6.04(1 \mathrm{H}, \mathrm{s}), 5.83(1 \mathrm{H}, \mathrm{t}, J 1.0), 2.94(2 \mathrm{H}, \mathrm{t}, J$ 7.0), $2.71(2 \mathrm{H}, \mathrm{t}, J 7.0), 2.37$ (2H, td, J 7.3, 1.0), $2.27(2 \mathrm{H}, \mathrm{t}, J 7.1), 2.06(3 \mathrm{H}, \mathrm{s}), 1.79$ $1.69(2 \mathrm{H}, \mathrm{m}) ; \delta_{\mathrm{C}}\left(75 \mathrm{MHz} ; \mathrm{CDCl}_{3}\right)$ 199.9, 146.9, 126.5, 119.8, 37.9, 30.6, 29.1, 24.7, 17.1, 16.3; m/z (ES+) 197 (10\%, M), 149 (40\%, M-MeSH) 75 (100\% M$\mathrm{COCCH}_{2}\left(\mathrm{CH}_{2}\right)_{3} \mathrm{CN}$; found $[\mathrm{M}+\mathrm{H}]^{+} 198.0945, \mathrm{C}_{10} \mathrm{H}_{16} \mathrm{NOS}$ requires 198.0945. 


\section{(E)-9-Methanesulfanyl-7-oxo-non-5-enenitrile, 7}<smiles>CSCCC(=O)C=CCCCC#N</smiles>

The general procedure for the hydroacylation reaction was followed employing precatalyst $[\mathrm{Rh}(\mathrm{nbd})(\mathrm{dppe})] \mathrm{ClO}_{4}(10 \mathrm{mg}, \quad 0.015 \mathrm{mmol})$, acetone $(2.0 \mathrm{~mL}), 3-$ methanesulfanyl propanal $(30 \mu \mathrm{L}, 0.3 \mathrm{mmol})$, MeCN $(12 \mu \mathrm{L}, 6.0 \mathrm{mmol})$ and 5hexynenitrile $(62 \mu \mathrm{L}, 0.6 \mathrm{mmol})$. Heated at $55^{\circ} \mathrm{C}$ for 4 hours. Flash chromatography (1:4, EtOAc:Petrol) yielded the title compound as a yellow oil (48 mg, $81 \%$ ); $v_{\max }$ (film) $/ \mathrm{cm}^{-1} 2908,2357,1665,1662,1428 ; \delta_{\mathrm{H}}\left(300 \mathrm{MHz} ; \mathrm{CDCl}_{3}\right) 6.73(1 \mathrm{H}, \mathrm{dt}, J$ 15.9, 6.9), 6.13 (1H, d, J 15.9), 2.82-2.68 (4H, m), 2.38-2.31 (4H, m), $2.06(3 \mathrm{H}, \mathrm{s}), 1.84-$ $1.75(2 \mathrm{H}, \mathrm{m}) ; \delta_{\mathrm{C}}\left(75 \mathrm{MHz} ; \mathrm{CDCl}_{3}\right)$ 196.8, 143.1, 130.1, 117.8, 39.0, 29.8, 27.0, 22.6, 15.5, 14.7; m/z (ES+) 197 (5 \%, M), 150 (40\%, M-MeS), 121 (100\%, M-( $\left.\left.\mathrm{CH}_{2}\right)_{3} \mathrm{CN}\right)$, $75\left(80 \% \mathrm{M}-\mathrm{COCCH}_{2}\left(\mathrm{CH}_{2}\right)_{3} \mathrm{CN}\right.$; found $\left[\mathrm{M}+\mathrm{NH}_{4}\right]^{+} 215.1213, \mathrm{C}_{10} \mathrm{H}_{19} \mathrm{~N}_{2} \mathrm{OS}$ requires 215.1213 .

\section{(E)-8-Methanesulfanyl-6-oxo-oct-4-enenitrile (a) and 4-(3-methanesulfanyl-} propionyl)-pent-4-enenitrile (b), Table 6 entry 1<smiles>CS(=O)CCC(=O)C=CCCC#N</smiles>

(a)<smiles>C=C(CCC#N)C(=O)CCS(C)(=O)=O</smiles>

(b)

The general procedure for the hydroacylation reaction was followed employing precatalyst $[\mathrm{Rh}(\mathrm{nbd})(\mathrm{dppe})] \mathrm{ClO}_{4}(10 \mathrm{mg}, \quad 0.015 \mathrm{mmol})$, acetone $(2.0 \mathrm{~mL}), 3-$ methanesulfanyl propanal $(30 \mu \mathrm{L}, 0.3 \mathrm{mmol}$ ) and 4-cyano-1-butyne (50 mg, 0.6 mmol). Heated at $55{ }^{\circ} \mathrm{C}$ for 1 hour. Flash chromatography (1:4, EtOAc:Petrol) yielded 8-methanesulfanyl-6-oxo-oct-4-enenitrile (a) as a pale yellow oil (25 mg, 46\%); $v_{\max }$ (film) $/ \mathrm{cm}^{-1} 3049,2295,1668,1411,1252,893,728 ; \delta_{\mathrm{H}}\left(300 \mathrm{MHz} ; \mathrm{CDCl}_{3}\right) 6.74(1 \mathrm{H}$, $\mathrm{dt}, J 15.9,6.4), 6.17$ (1H, d, $J 15.9), 2.82$ (2H, t, $J 6.5), 2.71(2 \mathrm{H}, \mathrm{t}, J 6.5), 2.57-2.46$ $(4 \mathrm{H}, \mathrm{m}), 2.06(3 \mathrm{H}, \mathrm{s}) ; \delta_{\mathrm{C}}\left(75 \mathrm{MHz} ; \mathrm{CDCl}_{3}\right)$ 196.6, 140.4, 130.6, 117.3, 39.3, 26.9, 26.8, 15.1, 14.7; m/z (EI) 183 (7\%, M), 135 (50\%, M-MeSH), 108 (90\%, M$\mathrm{MeSCH}_{2} \mathrm{CH}_{2}$ ); found $[\mathrm{M}]^{+}$183.0713, $\mathrm{C}_{9} \mathrm{H}_{13} \mathrm{NOS}$ requires 183.0712; and 4-(3methylsulfanyl-propionyl)-pent-4-enenitrile (b) as a pale yellow oil (17 $\mathrm{mg}, 31 \%$ ); $v_{\max }($ film $) / \mathrm{cm}^{-1} 3049,2295,1668,1411,1252,893,728 ; \delta_{\mathrm{H}}\left(300 \mathrm{MHz} ; \mathrm{CDCl}_{3}\right) 6.22$ $(1 \mathrm{H}, \mathrm{s}), 6.04(1 \mathrm{H}, \mathrm{s}), 3.06(2 \mathrm{H}, \mathrm{t}, J 7.3), 2.77(2 \mathrm{H}, \mathrm{t}, J 7.3), 2.63-2.52(4 \mathrm{H}, \mathrm{m}), 2.13$ 
$(3 \mathrm{H}, \mathrm{s}) ; \delta_{\mathrm{C}}\left(75 \mathrm{MHz} ; \mathrm{CDCl}_{3}\right)$ 198.5, 143.7, 127.2, 118.3, 36.7, 27.8, 26.9, 16.2, 15.2; $m / z$ (EI) 183 (7\%, M), 135 (50\%, M-MeSH), 108 (90\%, M-MeSCH $\left.\mathrm{CH}_{2}\right)$; found [M] ${ }^{+}$ 183.0714, $\mathrm{C}_{9} \mathrm{H}_{13} \mathrm{NOS}$ requires 183.0712

(E)-10-Methanesulfanyl-8-oxo-dec-6-enenitrile (a) and 6-(3-methanesulfanylpropionyl)-hept-6-enenitrile (b), Table 6 entry 2<smiles>CSCCC(=O)C=CCCCCC#N</smiles>

(a)<smiles>C=C(CCCCC#N)C(=O)CCS(C)(=O)=O</smiles>

(b)

The general procedure for the hydroacylation reaction was followed employing precatalyst $[\mathrm{Rh}(\mathrm{nbd})(\mathrm{dppe})] \mathrm{ClO}_{4}(10 \mathrm{mg}, \quad 0.015 \mathrm{mmol})$, acetone $(2.0 \mathrm{~mL}), 3-$ methanesulfanyl propanal $(30 \mu \mathrm{L}, 0.3 \mathrm{mmol})$ and hept-6-ynenitrile ${ }^{3}$ (64 mg, 0.6 mmol). Heated at $55{ }^{\circ} \mathrm{C}$ for 1 hour. Flash chromatography (1:4, EtOAc:Petrol) yielded 10-methanesulfanyl-8-oxo-dec-6-enenitrile (a) as a pale yellow oil (31 mg, 49\%); $v_{\max }$ (film) $/ \mathrm{cm}^{-1} 3049,2978,1673,1416,1252,887,744 ; \delta_{\mathrm{H}}\left(300 \mathrm{MHz} ; \mathrm{CDCl}_{3}\right) 6.76$ (1H, dt, $J 15.9,6.9), 6.08$ (1H, dt, $J 15.9,1.5), 2.82-2.68(4 \mathrm{H}, \mathrm{m}), 2.32$ (2H, t, $J 6.7)$, 2.26-2.19 (2H, m), $2.06(3 \mathrm{H}, \mathrm{s}), 1.69-1.56(4 \mathrm{H}, \mathrm{m}) ; \delta_{\mathrm{C}}\left(75 \mathrm{MHz} ; \mathrm{CDCl}_{3}\right)$ 197.6, 145.4, 130.0, 118.7, 39.3, 30.8, 27.6, 26.3, 24.2, 16.4, 15.2; m/z (ES+) $211(5 \%, \mathrm{M}+), 164$ (30\%, M-MeS), 74 (80\%, M- $\left.\mathrm{CN}\left(\mathrm{CH}_{2}\right)_{4} \mathrm{CHCHCOH}\right), 55\left(100 \%, \mathrm{M}_{-} \mathrm{CH}_{3} \mathrm{CH}_{2} \mathrm{CN}\right)$; found 212.1099 [M+H] $]^{+} \mathrm{C}_{11} \mathrm{H}_{17} \mathrm{NOS}$ requires 212.1104; and 6-(3-methanesulfanylpropionyl)-hept-6-enenitrile (b) as a colourless oil $(20 \mathrm{mg}, 32 \%) ; v_{\max }(\mathrm{film}) / \mathrm{cm}^{-1}$ 3048, 2978, 1650, 1420, 1235, 900; $\delta_{\mathrm{H}}\left(300 \mathrm{MHz} ; \mathrm{CDCl}_{3}\right) 6.06(1 \mathrm{H}, \mathrm{s}), 5.82(1 \mathrm{H}, \mathrm{s})$, $3.00(2 \mathrm{H}, \mathrm{t}, J 6.9), 2.77$ (2H, t, J 6.9), 2.42-2.26 (4H, m), $2.13(3 \mathrm{H}, \mathrm{s}), 1.70-1.54(4 \mathrm{H}$, $\mathrm{m}) ; \delta_{\mathrm{C}}\left(75 \mathrm{MHz} ; \mathrm{CDCl}_{3}\right) 200.2,148.1,125.5,120.0,38.0,30.4,29.1,28.0,25.5,17.4$, 16.3; $m / z \quad(\mathrm{ES}+) \quad 211 \quad(5 \%, \quad \mathrm{M}+), \quad 164 \quad(30 \%, \quad \mathrm{M}-\mathrm{MeS}), \quad 74 \quad(80 \%, \quad \mathrm{M}-$ $\left.\mathrm{CN}\left(\mathrm{CH}_{2}\right)_{4} \mathrm{CHCHCOH}\right), 55\left(100 \%, \quad \mathrm{M}-\mathrm{CH}_{3} \mathrm{CH}_{2} \mathrm{CN}\right) ;$ found $212.1099[\mathrm{M}+\mathrm{H}]^{+}$ $\mathrm{C}_{11} \mathrm{H}_{17} \mathrm{NOS}$ requires 212.1104 .

\footnotetext{
${ }^{3}$ Davison, E. C.; Fox, M. E.; Holmes, A. B.; Roughley, S. D.; Smith, C. J.; Williams, G. M.; Davies, J. E.; Raithby, P. R.; Adams, J. P.; Forbes, I. T.; Press, N. J.; Thompson, M. J. J. Chem. Soc., Perkin Trans. 1 2002, 1494-1514.
} 
(E)-4-Methyl-1-methanesulfanyl-dec-4-en-3-one (a) and (E)-4-Ethylidene-1methanesulfonyl-nonan-3-one (b), Table 6 entry 3<smiles>CCCCC/C=C(\C)C(=O)CCS(C)(=O)=O</smiles>

(a)<smiles>CC=C(CCCCC)C(=O)CCSC</smiles>

(b)

The general procedure for the hydroacylation reaction was followed employing precatalyst $\left[\mathrm{Rh}(\mathrm{nbd})(\mathrm{dppe}) \mathrm{ClO}_{4} \quad(10 \mathrm{mg}, \quad 0.015 \mathrm{mmol})\right.$, acetone $(2 \mathrm{~mL}), 3-$ methanesulfanyl propanal $(30 \mu \mathrm{L}, 0.3 \mathrm{mmol})$ and 2-octyne $(87 \mu \mathrm{L}, 0.6 \mathrm{mmol})$. Heated at $55{ }^{\circ} \mathrm{C}$ for 48 hours. Flash chromatography (1:4, EtOAc:Petrol) yielded the title compounds as a colourless oil as a $3: 1$ mixture of $\mathbf{a}$ and $\mathbf{b}(37 \mathrm{mg}, 58 \%) ; v_{\max }$ (film) $/ \mathrm{cm}^{-1}$ 2921, 2854, 2253, 1663, 1468, 1375, 903, 723; $\delta_{\mathrm{H}}\left(300 \mathrm{MHz} ; \mathrm{CDCl}_{3}\right)$ (major a) $6.58(1 \mathrm{H}, \mathrm{td}, J 7.2,1.2), 2.93-2.88(2 \mathrm{H}, \mathrm{m}), 2.73-2.67(2 \mathrm{H}, \mathrm{m}), 2.24-2.14$ $(2 \mathrm{H}, \mathrm{m}), 2.06(3 \mathrm{H}, \mathrm{s}), 1.71(3 \mathrm{H}$, br. s), 1.45-1.36 (2H, m), 1.28-1.20 (4H, m), 0.86$0.78(3 \mathrm{H}, \mathrm{m})$; (minor b) $\delta_{\mathrm{H}}\left(300 \mathrm{MHz} ; \mathrm{CDCl}_{3}\right) 6.66(1 \mathrm{H}, \mathrm{q}, J 7.0), 2.93-2.88(2 \mathrm{H}, \mathrm{m})$, 2.73-2.67 (2H, m), 2.24-2.14 (2H, m), $2.05(3 \mathrm{H} \mathrm{s}), 1.76(3 \mathrm{H}, \mathrm{d}, J 7.0), 1.45-1.36(6 \mathrm{H}$, $\mathrm{m}) ; \delta_{\mathrm{C}}\left(75 \mathrm{MHz} ; \mathrm{CDCl}_{3}\right)$ (mixture) 199.6, 199.3, 143.2, 142.9, 137.4, 136.8, 37.0, $36.9,31.7,31.4,28.9,28.8,28.4,28.1,25.1,22.3,22.2$, 15.7, 14.5, 13.83, 13.78, 11.1; $m / z(\mathrm{EI}+) 214(20 \%, \mathrm{M}+), 166(95 \%, \mathrm{MeSH}), 109\left(100 \%, \mathrm{MeS}\left(\mathrm{CH}_{2}\right)_{2} \mathrm{COH}\right)$; found 214.1385 [M] $]^{+} \mathrm{C}_{12} \mathrm{H}_{22} \mathrm{OS}$ requires 214.1386.

\section{Hept-5-ynenitrile, Table 6 entry 4 substrate}

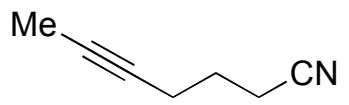

6-Chlorohex-2-yne $(1.16 \mathrm{~g}, 0.01 \mathrm{~mol})$ was added to a solution of $\mathrm{NaCN}(0.5 \mathrm{~g}, 0.011$ $\mathrm{mol})$ in $\mathrm{EtOH}(10.5 \mathrm{~mL})$ and water $(3 \mathrm{~mL})$. The resulting solution was heated at reflux for 16 hours. It was then cooled to room temperature diluted with DCM $(50 \mathrm{~mL})$. The solution was extracted with water $(3 \times 50 \mathrm{~mL})$ and dried $\left(\mathrm{MgSO}_{4}\right)$. After this time the solution was reduced in vacu and purified by flash chromatography (1:9 EtOAc:Petrol) giving the title compound as a colourless oil $(0.95 \mathrm{~g}, 89 \%) ; v_{\max }($ film) $/ \mathrm{cm}^{-1}$ 2921, 2243, 1422, 1267, 749; $\delta_{\mathrm{H}}\left(300 \mathrm{MHz} ; \mathrm{CDCl}_{3}\right) 2.42$ (2H, t, J 7.2), 2.28$2.21(2 \mathrm{H}, \mathrm{m}), 1.81-1.74(2 \mathrm{H}, \mathrm{m}), 1.72(3 \mathrm{H}, \mathrm{t}, J 2.5) ; \delta_{\mathrm{C}}\left(75 \mathrm{MHz} ; \mathrm{CDCl}_{3}\right)$ 119.3, 77.6, 76.2, 24.8, 17.8, 16.0, 3.4; m/z (EI) 107 (10\%, M), 106 (100\%, M-H), 79 (40\%, M- 
$\left.\mathrm{H}_{2} \mathrm{CN}\right), 53\left(50 \%, \mathrm{M}-\mathrm{CH}_{3} \mathrm{CCCH}_{2}\right), 41\left(70 \%, \mathrm{M}-\mathrm{CNCH}_{2} \mathrm{CH}_{2} \mathrm{C}\right)$; found $[\mathrm{M}-\mathrm{H}]^{+}$ 106.0651, $\mathrm{C}_{7} \mathrm{H}_{9} \mathrm{~N}$ requires 106.0651 .

(E)-5-(3-Methanesulfanyl-propionyl)-hept-5-enenitrile (a) and (E)-6-methyl-9methanesulfanyl-7-oxo-non-5-enenitrile (b), Table 6 entry 4<smiles>CC=CC(=O)CCCC#N</smiles><smiles>CSCCC(=O)/C(C)=C/CCCC#N</smiles>

(b)

The general procedure for the hydroacylation reaction was followed employing precatalyst $\left[\mathrm{Rh}(\mathrm{nbd})(\mathrm{dppe}) \mathrm{ClO}_{4} \quad(10 \mathrm{mg}, \quad 0.015 \mathrm{mmol})\right.$, acetone $(2 \mathrm{~mL}), 3-$ methanesulfanyl propanal $(30 \mu \mathrm{L}, 0.3 \mathrm{mmol})$ and hept-5-ynenitrile $(64 \mathrm{mg}, 0.6$ mmol). Heated at $55{ }^{\circ} \mathrm{C}$ for 16 hours. Flash chromatography (1:4, EtOAc:Petrol) yielded 5-(3-Methanesulfanyl-propionyl)-hept-5-enenitrile (a) (33 mg, $53 \%$ ); $v_{\max }$ (film) $/ \mathrm{cm}^{-1} 2926,1673,1432,1252,733 ; \delta_{\mathrm{H}}\left(300 \mathrm{MHz} ; \mathrm{CDCl}_{3}\right) 6.85(1 \mathrm{H}, \mathrm{q}, J 7.0)$, $2.95(2 \mathrm{H}, \mathrm{t}, J 6.8), 2.76(2 \mathrm{H}, \mathrm{t}, J 6.8), 2.45(2 \mathrm{H}, \mathrm{t}, J 7.6), 2.32(2 \mathrm{H}, \mathrm{t}, J 7.1), 2.32(2 \mathrm{H}$, t, $J, 7.1), 2.12(3 \mathrm{H}, \mathrm{s}), 1.94(3 \mathrm{H}, \mathrm{d}, J 7.0), 1.76-1.66(2 \mathrm{H}, \mathrm{m}) ; \delta_{\mathrm{C}}\left(75 \mathrm{MHz} ; \mathrm{CDCl}_{3}\right)$ 199.0, 140.6, 139.6, 119.5, 36.7, 28.8, 24.3, 24.0, 16.8, 15.7, 14.7; m/z (EI) $211(10 \%$, M), $162\left(100 \%, \mathrm{M}-\mathrm{CH}_{3} \mathrm{SH}_{2}\right), 108\left(80 \%, \mathrm{M}-\mathrm{CH}_{3} \mathrm{CHC}\left(\mathrm{CH}_{2}\right)_{3} \mathrm{CN}\right), 75$ (50\%, M$\mathrm{COCH}_{3} \mathrm{CHC}\left(\mathrm{CH}_{2}\right)_{3} \mathrm{CN}$ ); found [M] 211.1024, $\mathrm{C}_{11} \mathrm{H}_{17} \mathrm{NOS}$ requires 211.1025; and 6methyl-9-methanesulfanyl-7-oxo-non-5-enenitrile (b) (17 mg, 27\%); $v_{\max }$ (film) / $\mathrm{cm}^{-1}$ 2920, 1650, 1454, 1234, 722; $\delta_{\mathrm{H}}\left(300 \mathrm{MHz} ; \mathrm{CDCl}_{3}\right) 6.56(1 \mathrm{H}, \mathrm{td}, J$ 7.2, 1.3$), 2.96$ (2H, t, J 7.1), $2.77(2 \mathrm{H}, \mathrm{t}, J 7.1), 2.47-2.38(4 \mathrm{H}, \mathrm{m}), 2.13(3 \mathrm{H}, \mathrm{s}), 1.91-1.81(5 \mathrm{H}, \mathrm{m})$; $\delta_{\mathrm{C}}\left(75 \mathrm{MHz} ; \mathrm{CDCl}_{3}\right) 199.3,138.8,138.6,118.8,37.1,28.7,27.5,24.2,16.6,15.7$, $11.4 ; \quad m / z \quad(\mathrm{EI}) 211 \quad(5 \%, \quad \mathrm{M}), 162 \quad\left(100 \%, \quad \mathrm{M}-\mathrm{CH}_{3} \mathrm{SH}_{2}\right), \quad 108 \quad(80 \%, \quad \mathrm{M}-$ $\left.\mathrm{CH}_{3} \mathrm{CHC}\left(\mathrm{CH}_{2}\right)_{3} \mathrm{CN}\right), 75$ (40\%, M-COCH$\left.{ }_{3} \mathrm{CHC}\left(\mathrm{CH}_{2}\right)_{3} \mathrm{CN}\right)$; found [M] 211.1023, $\mathrm{C}_{11} \mathrm{H}_{17} \mathrm{NOS}$ requires 211.1025 . 
Methyl 6-ethylsulfanyl-4-oxo-hexanoate, Table 3 entry 1

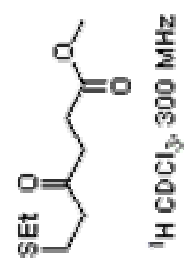

xanoate, Table 3 entry 1

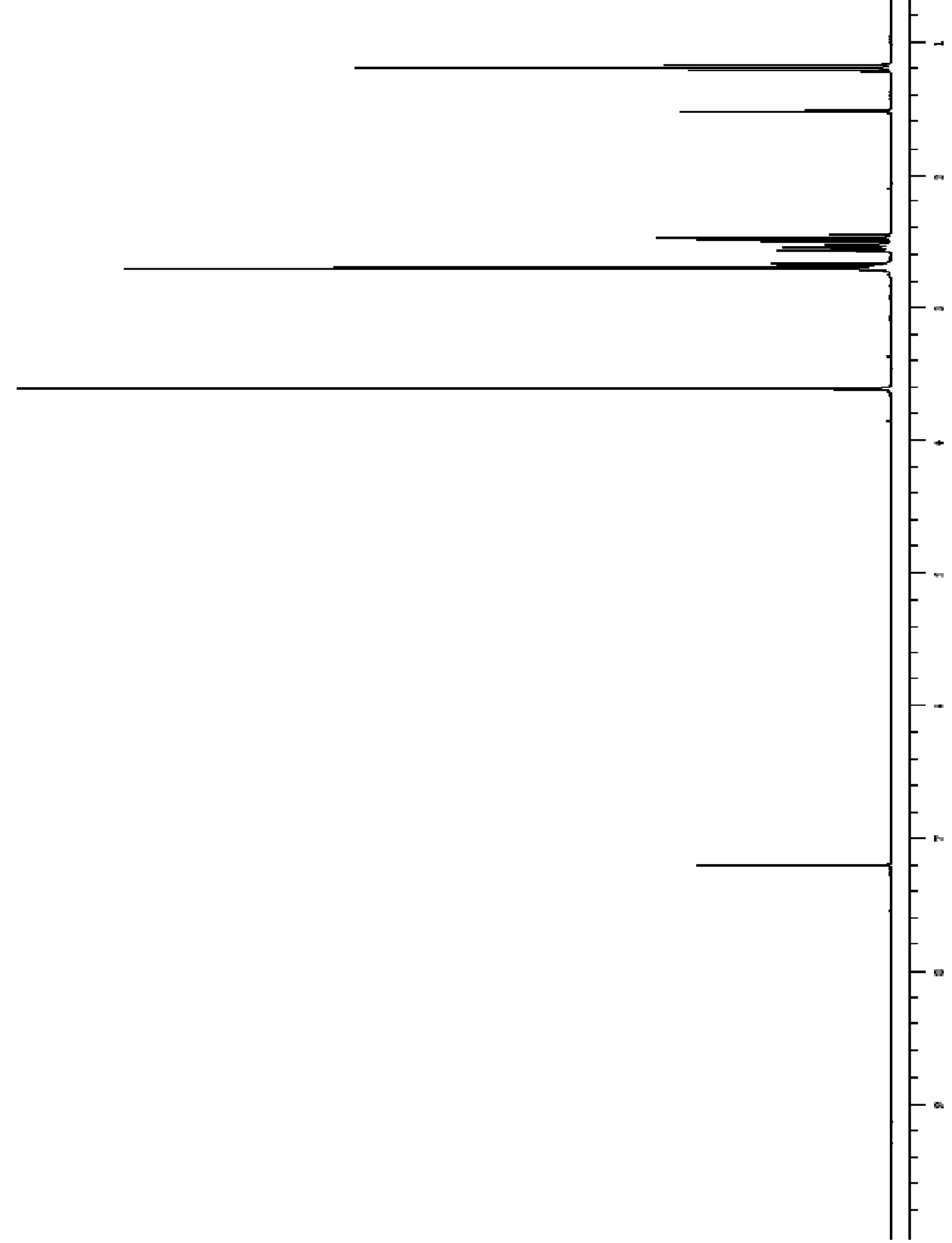


Methyl 4-oxo-6-phenylsulfanyl-hexanoate, Table 3 entry 2
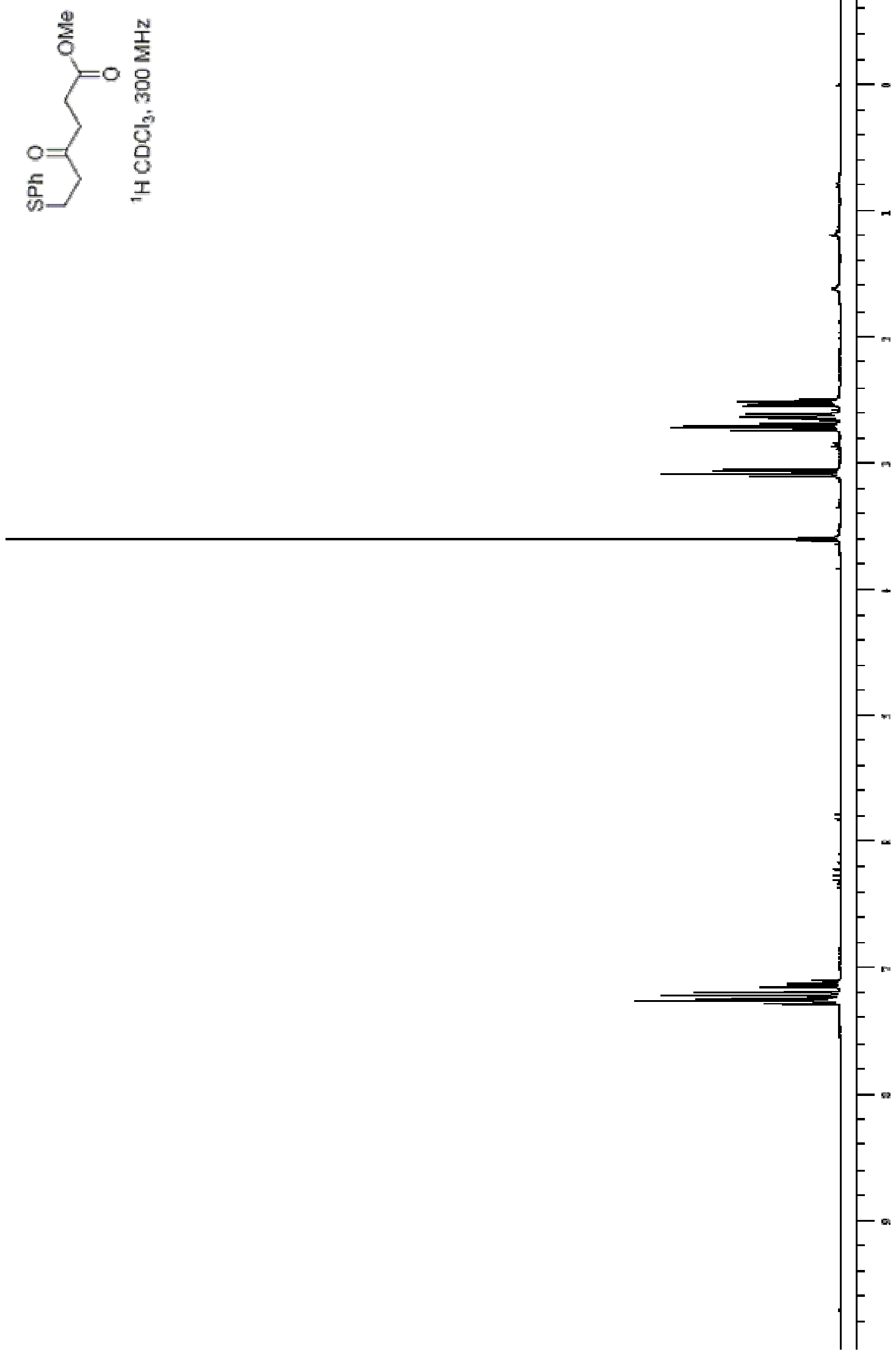


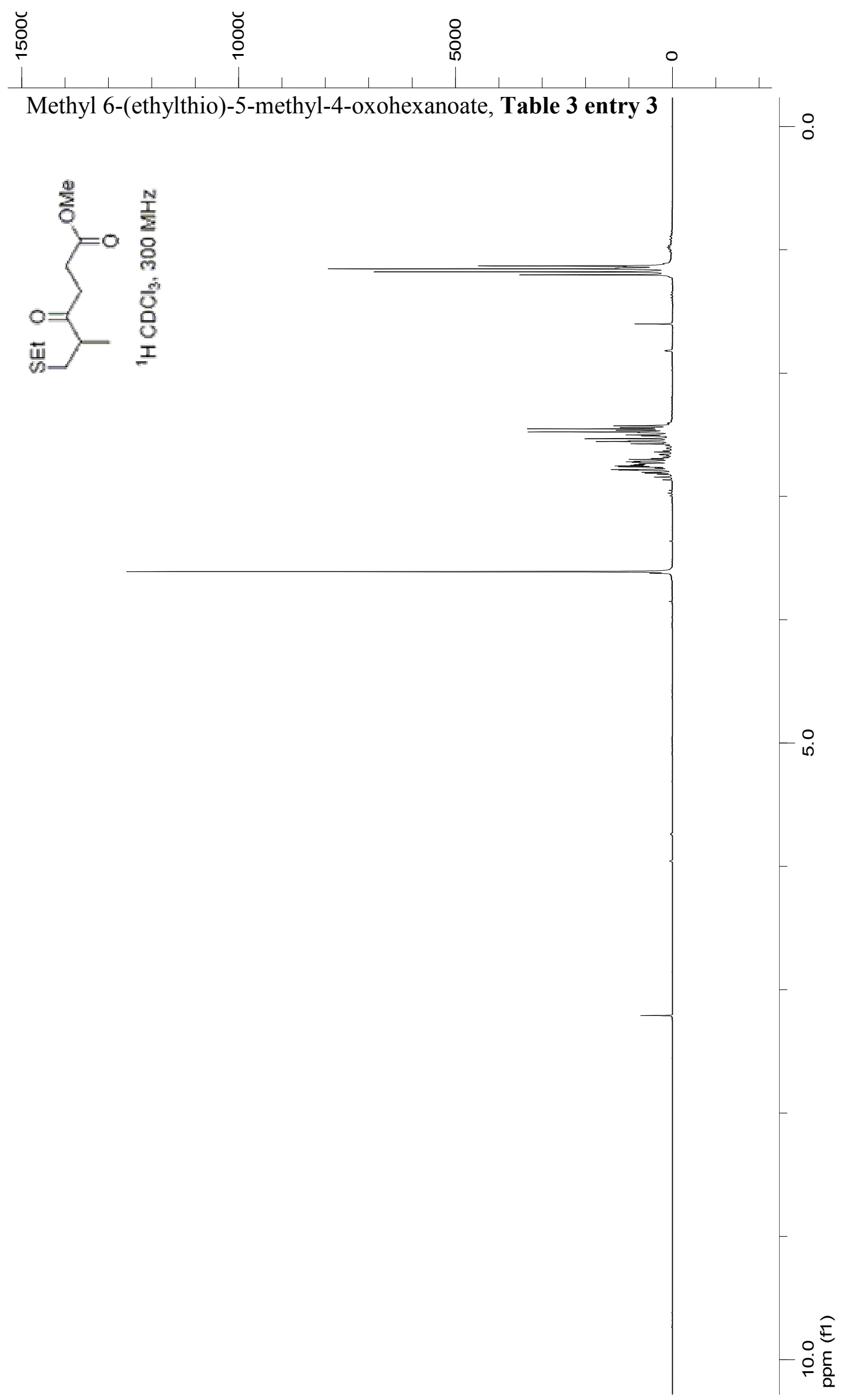




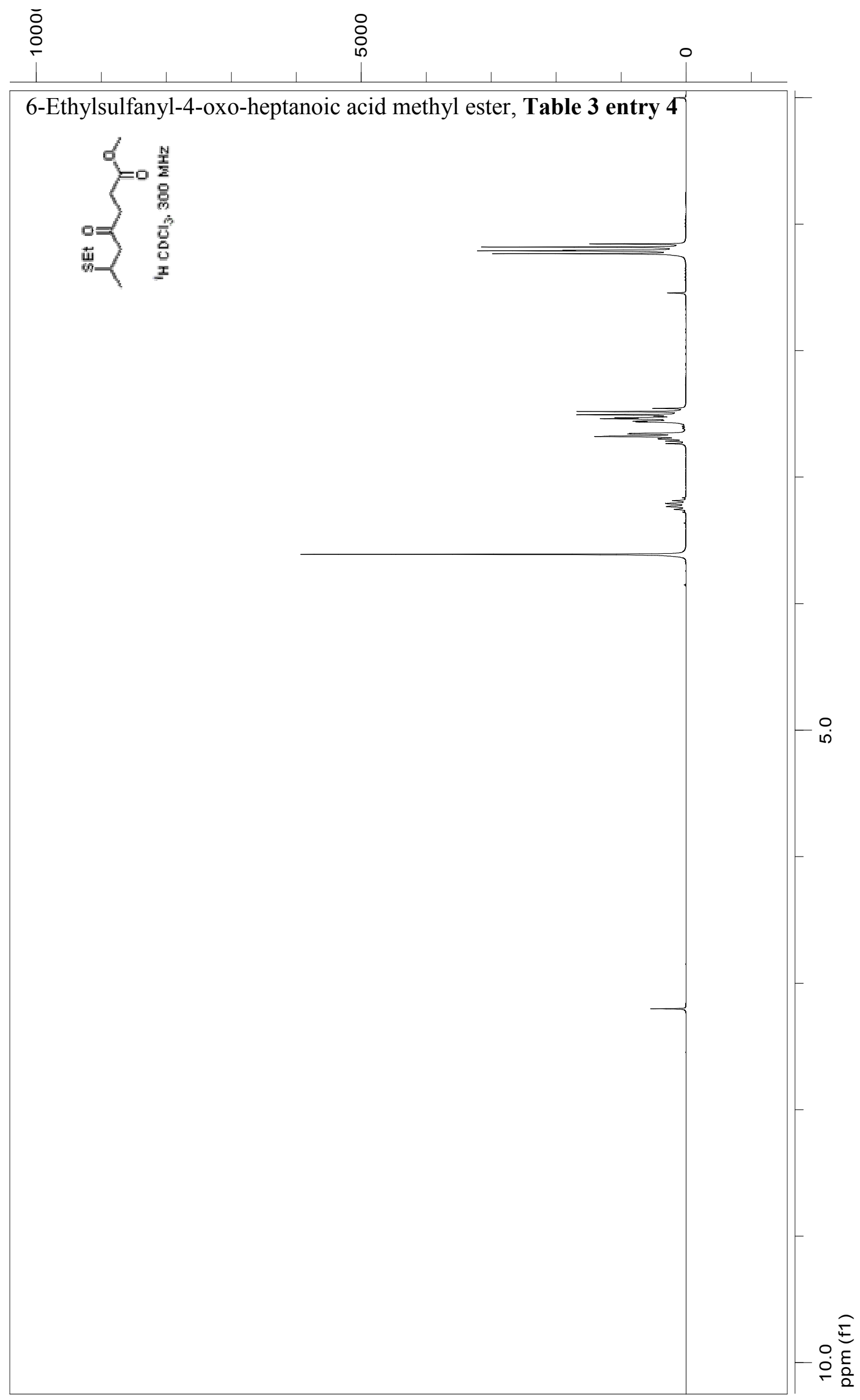




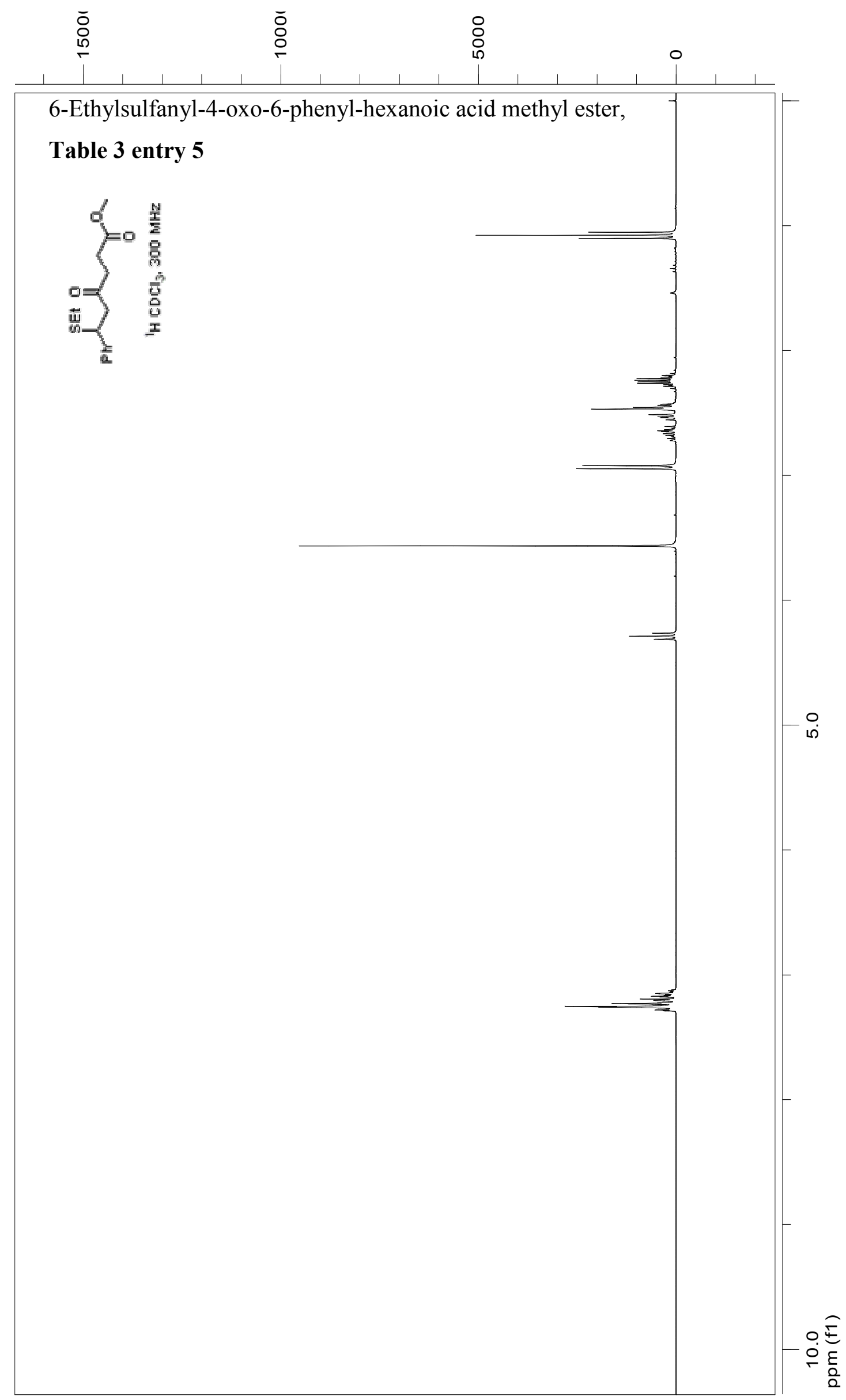


Methyl 6-ethylsulfanyl-5-methyl-4-oxo-heptanoate, Table 3 entry 6
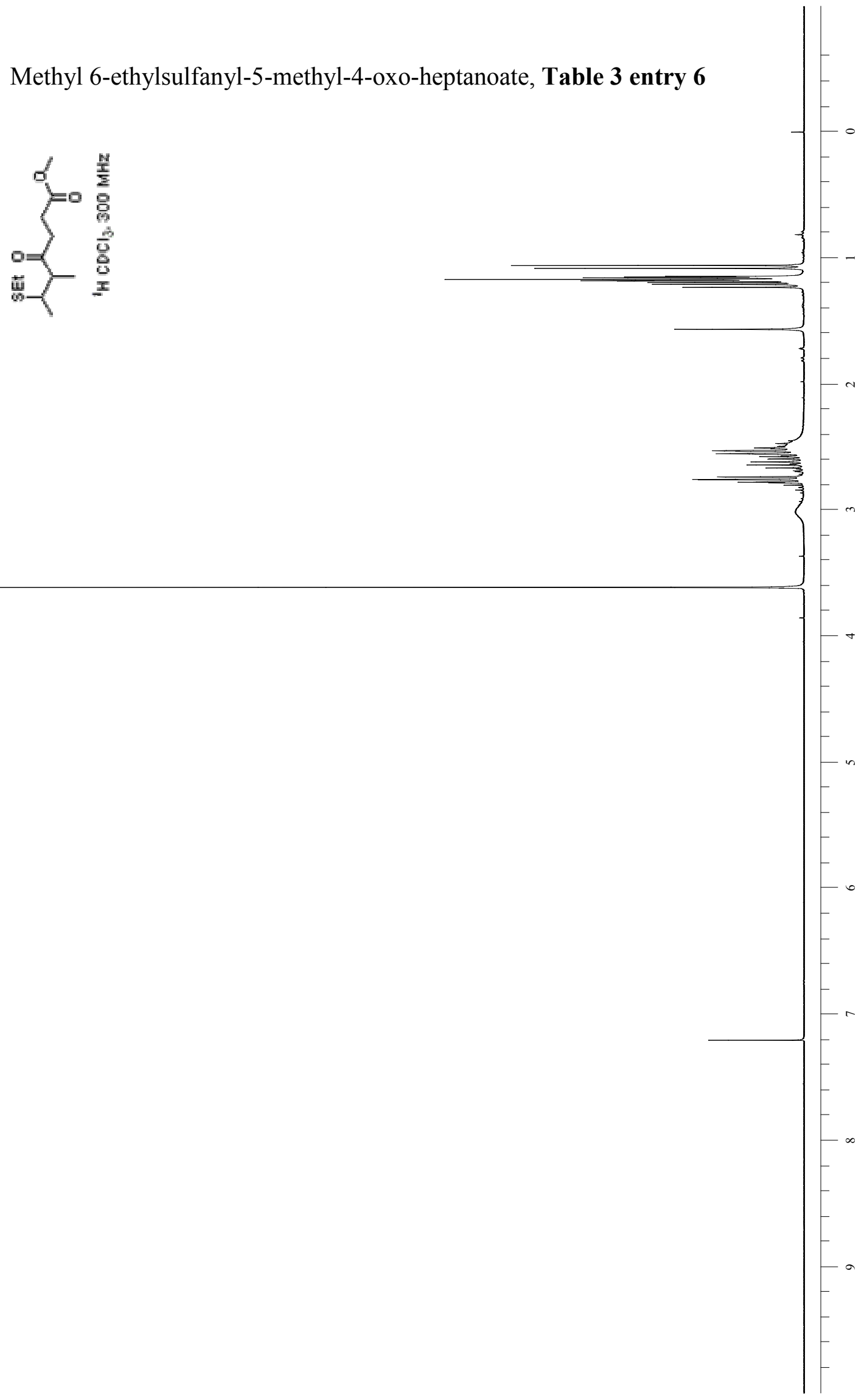


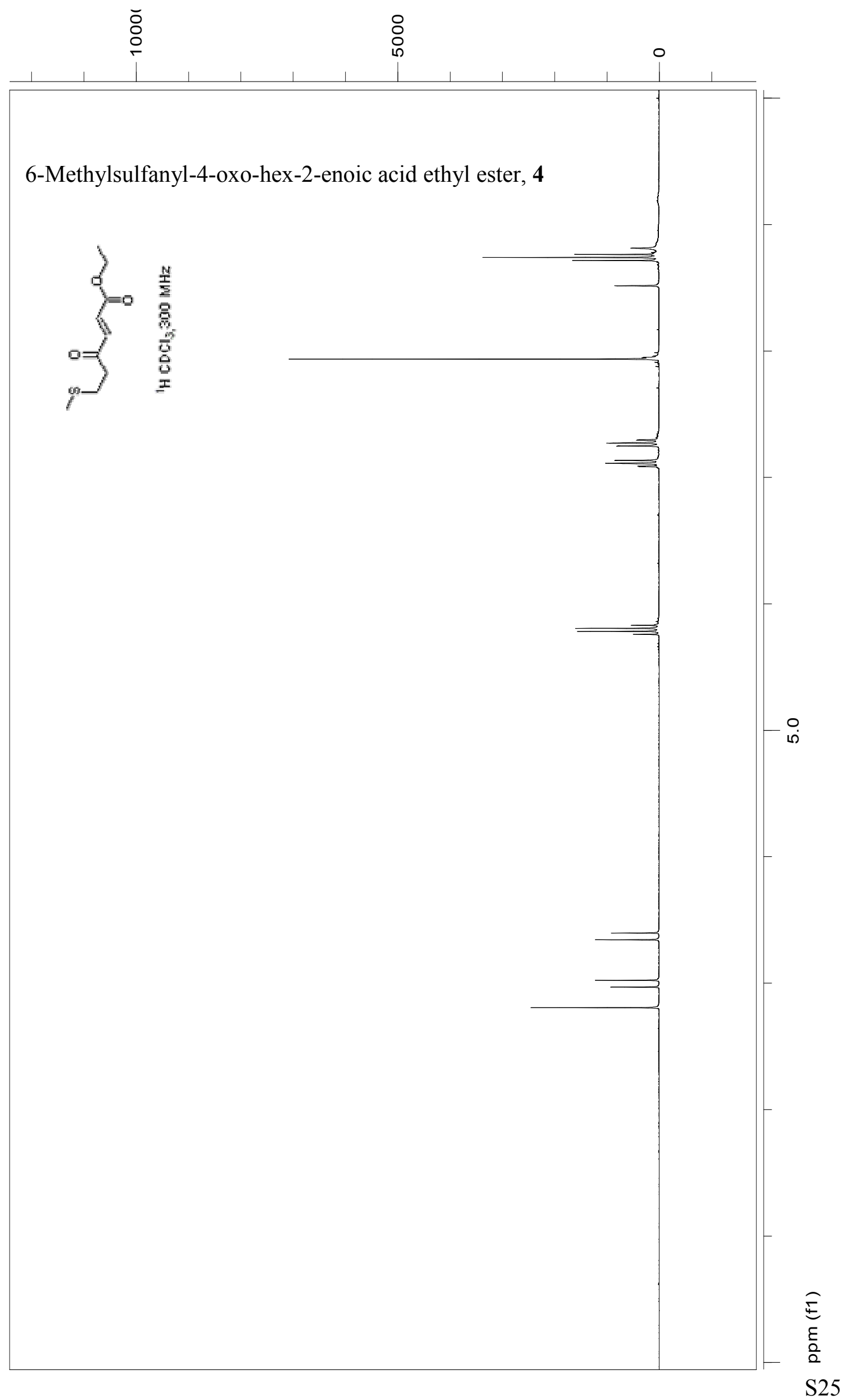




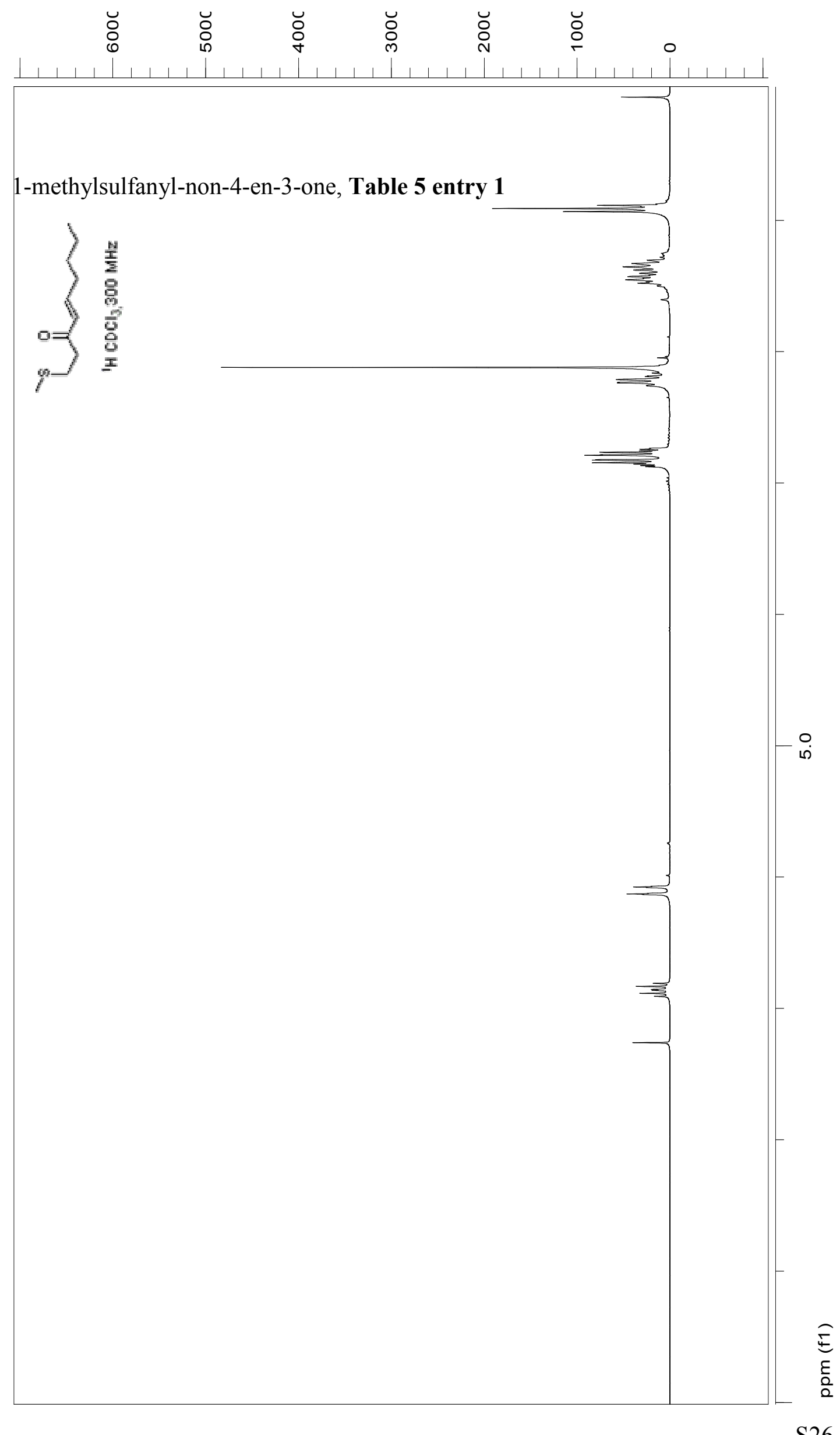




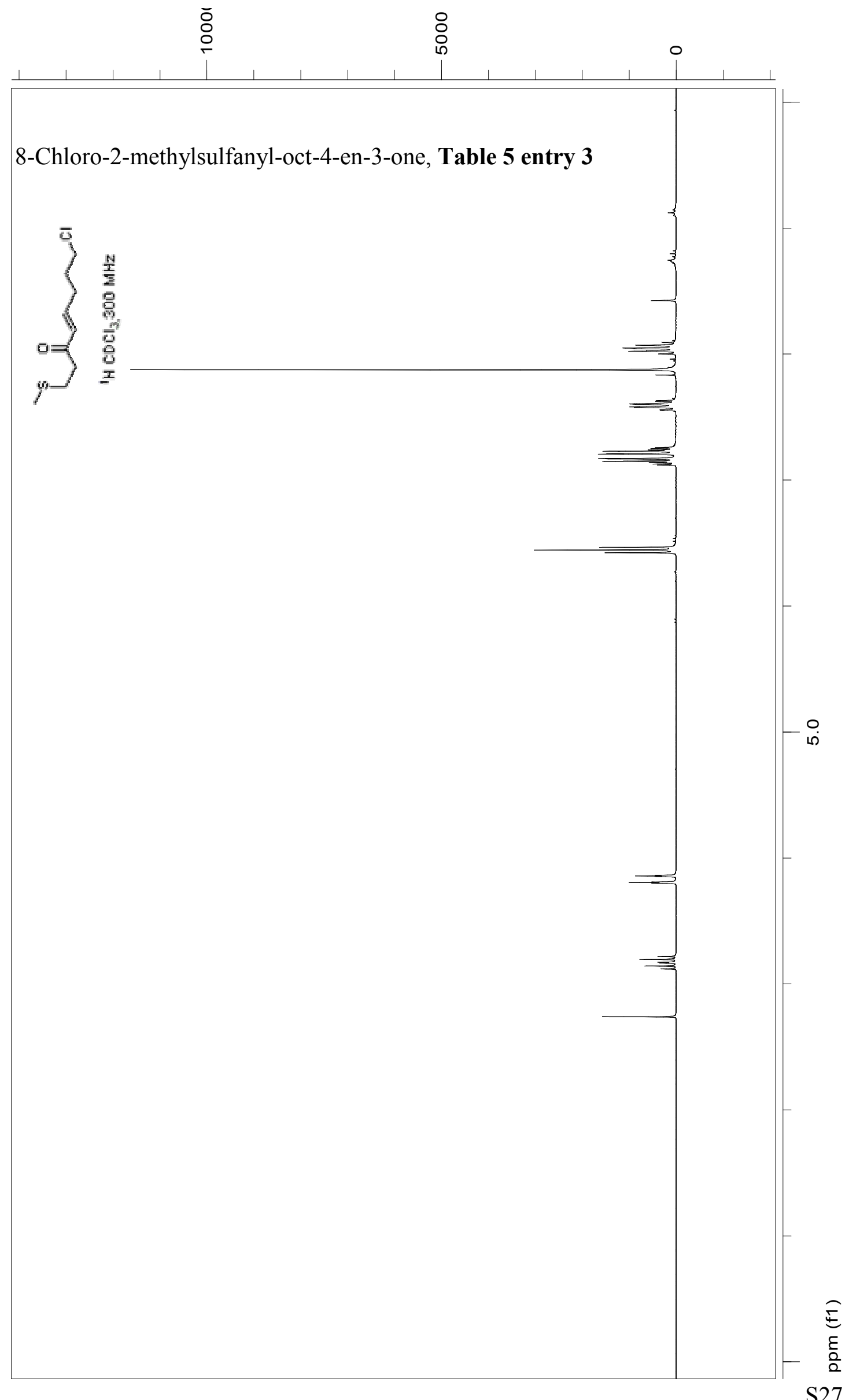




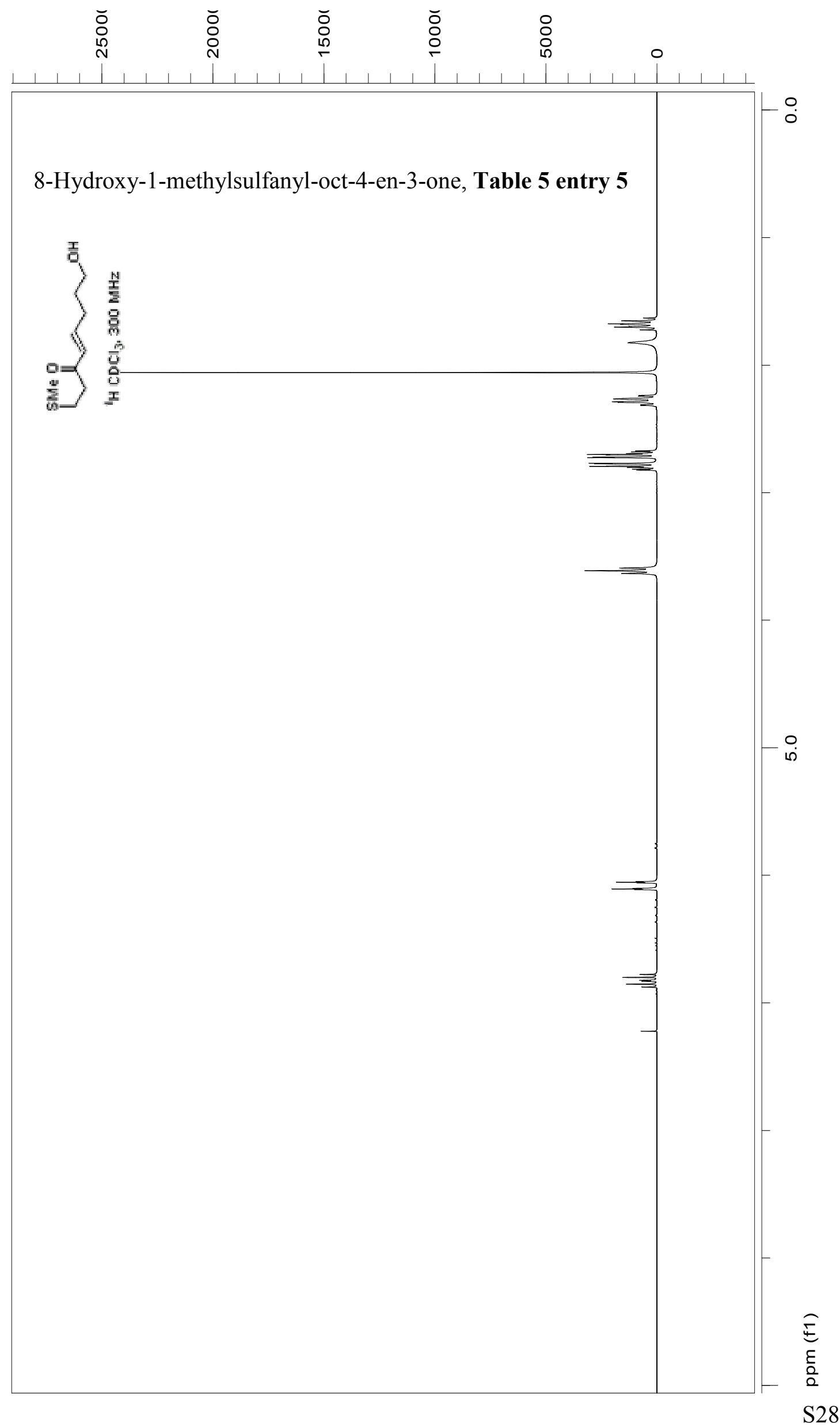




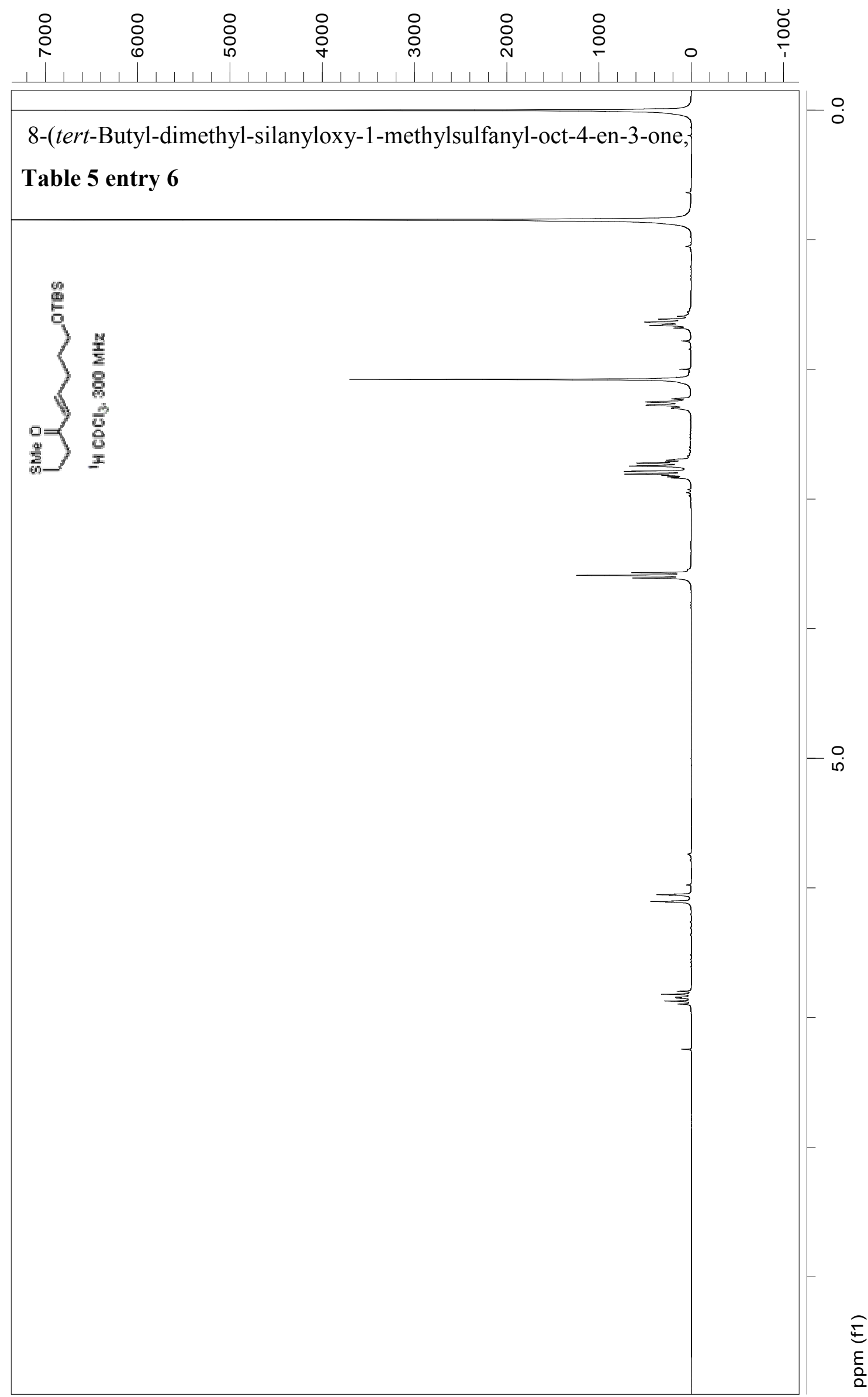

$\underset{E}{E}$
$\frac{2}{2}$ 


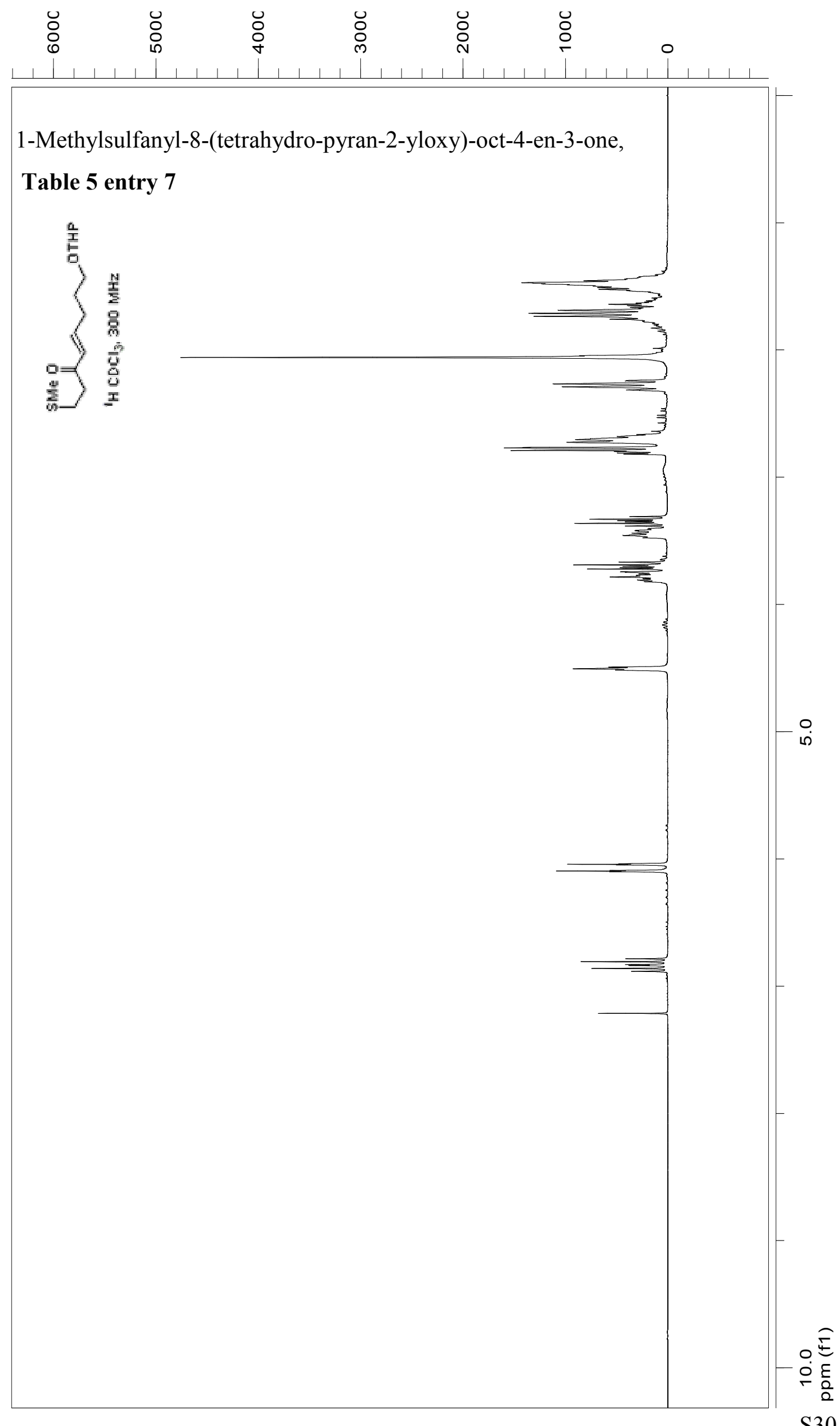



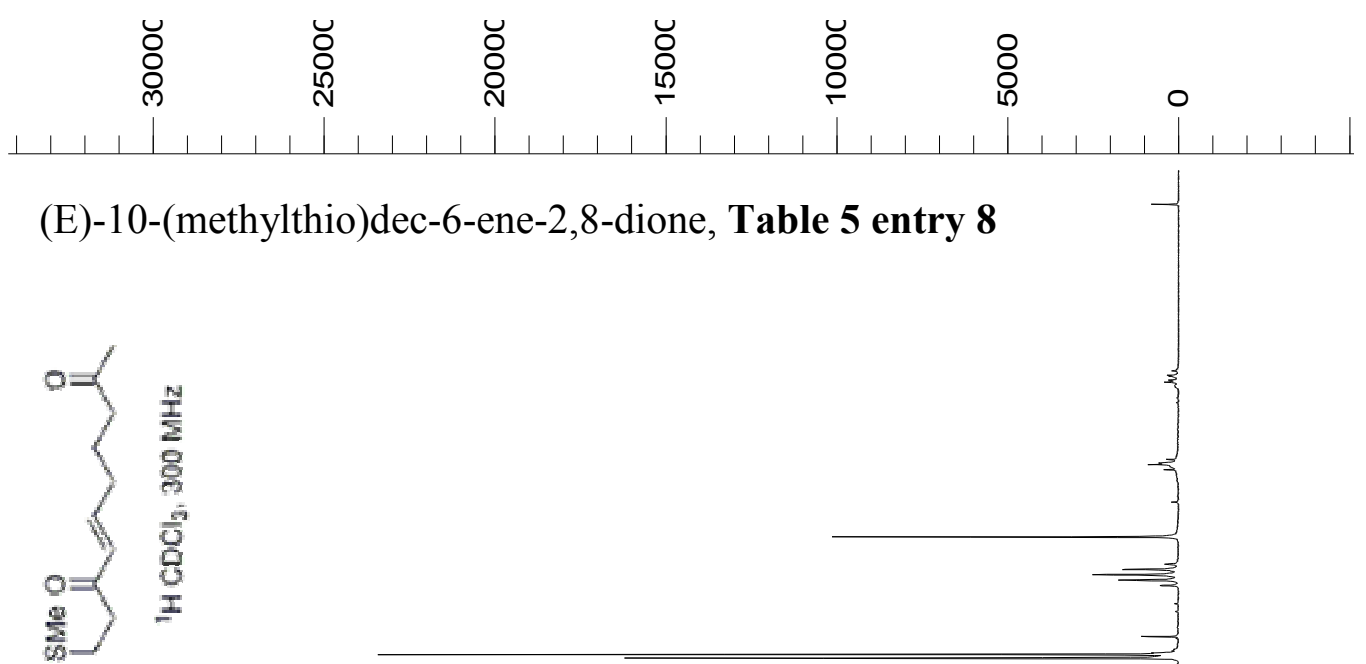

dec-6-ene-2,8-dione, Table 5 entry 8

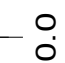




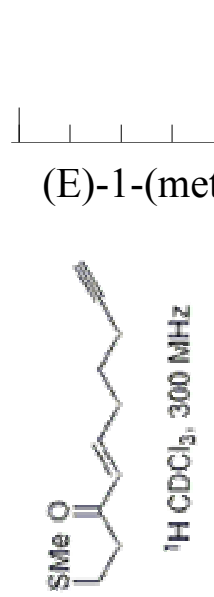

ญัญ

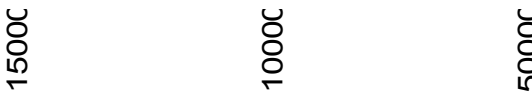

ఫั

(E)-1-(methylthio)dec-4-en-9-yn-3-one, Table 5 entry 9

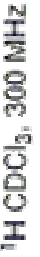




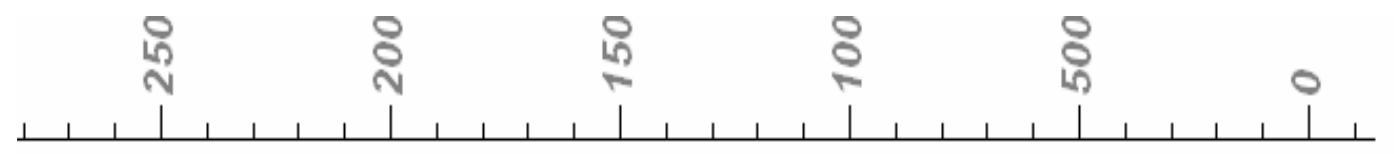

(4E,9E)-1,13-bis(methylthio)trideca-4,9-diene-3,11-dione,

Table 5 entry 10
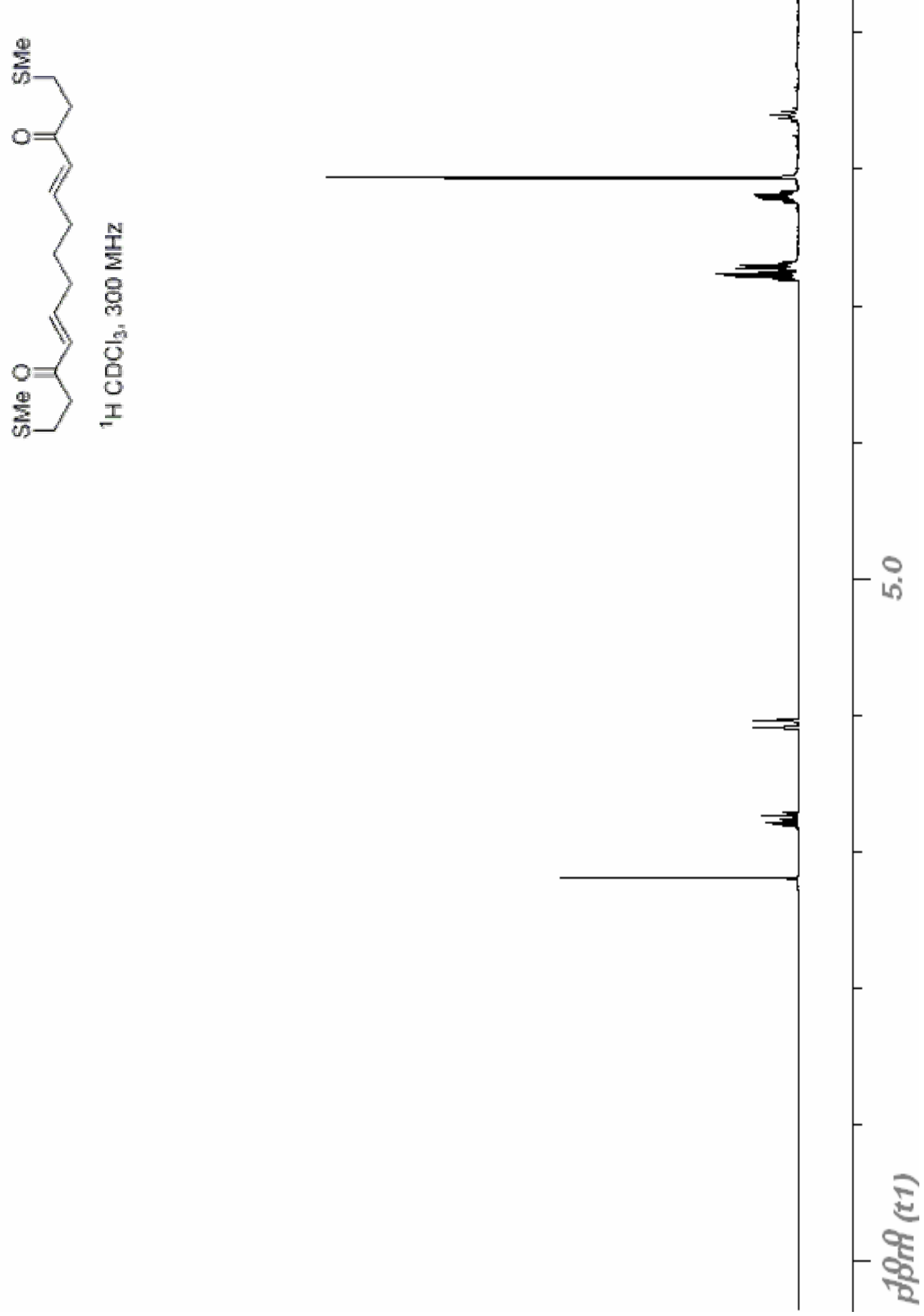


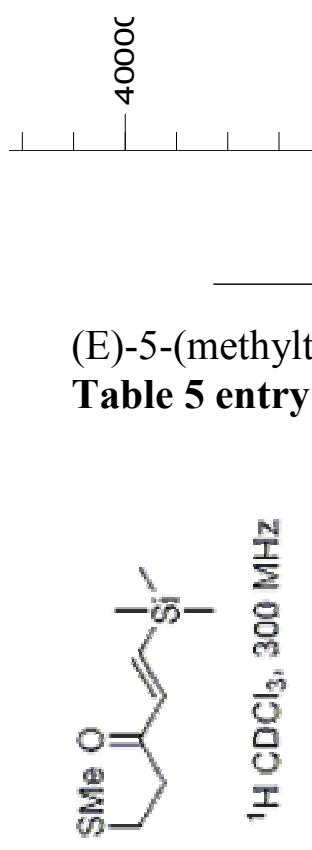

E)-5-(methylthio)-1-trimethylsilyl)pent-1-en-3-one,

Table 5 entry 11 


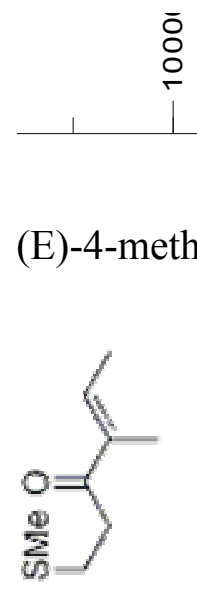

훙

응

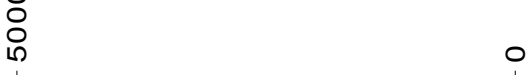

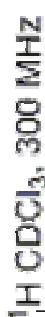




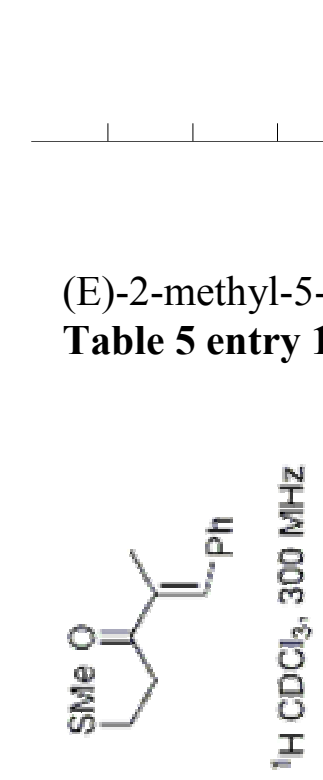

8

ethylthio)-1-phenylpent-1-en-3-one,

Table 5 entry 13

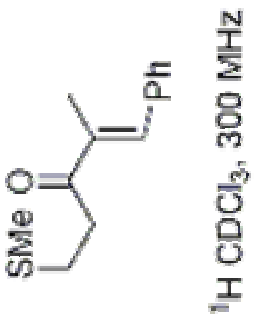


<smiles>COB(O)O[Mg]</smiles>

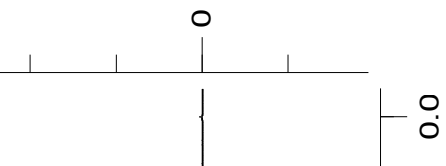

(E)-6-(methylthio)-1,3-diphenylhex-2-ene-1,4-dione, Table 5 entry 14 


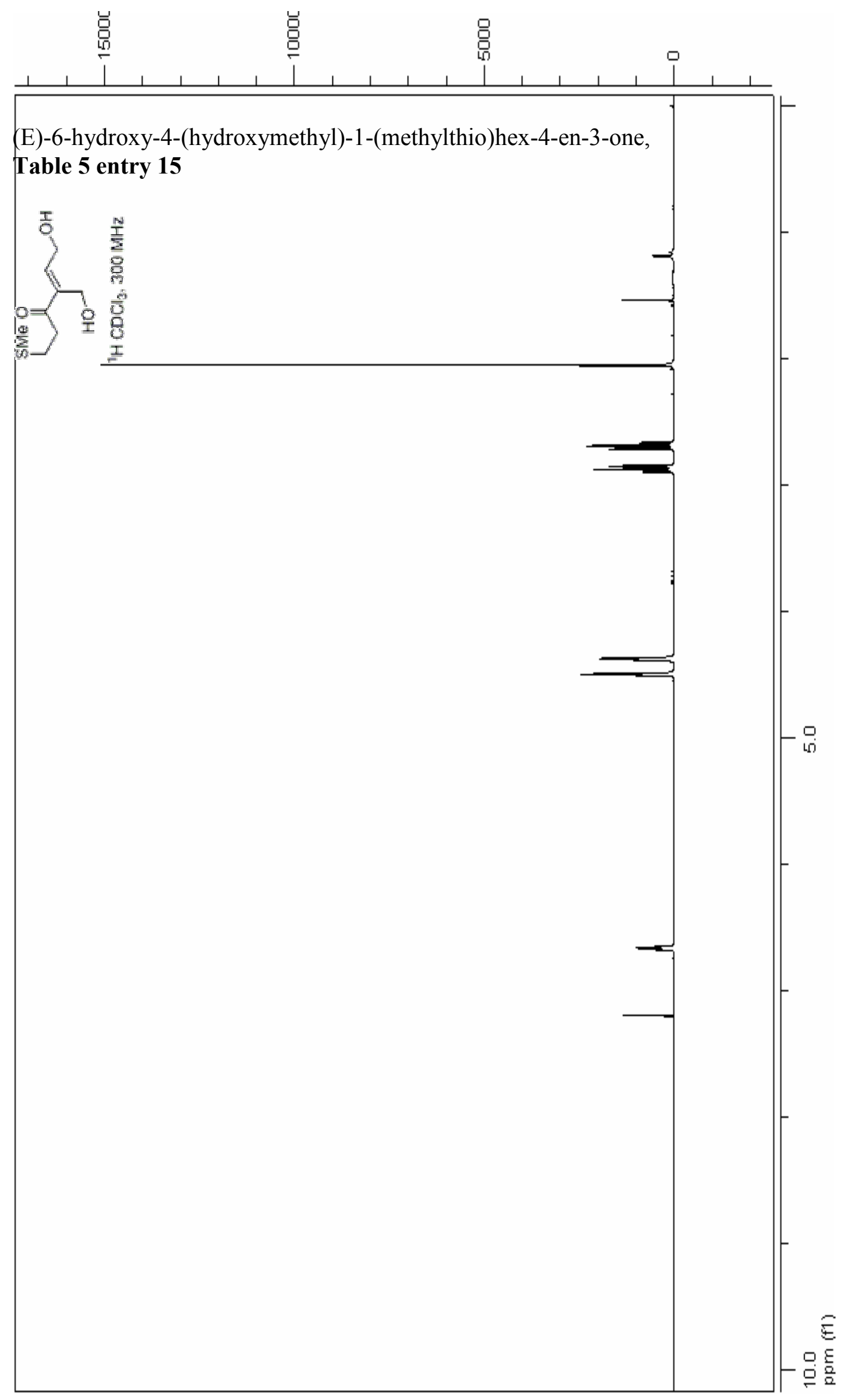



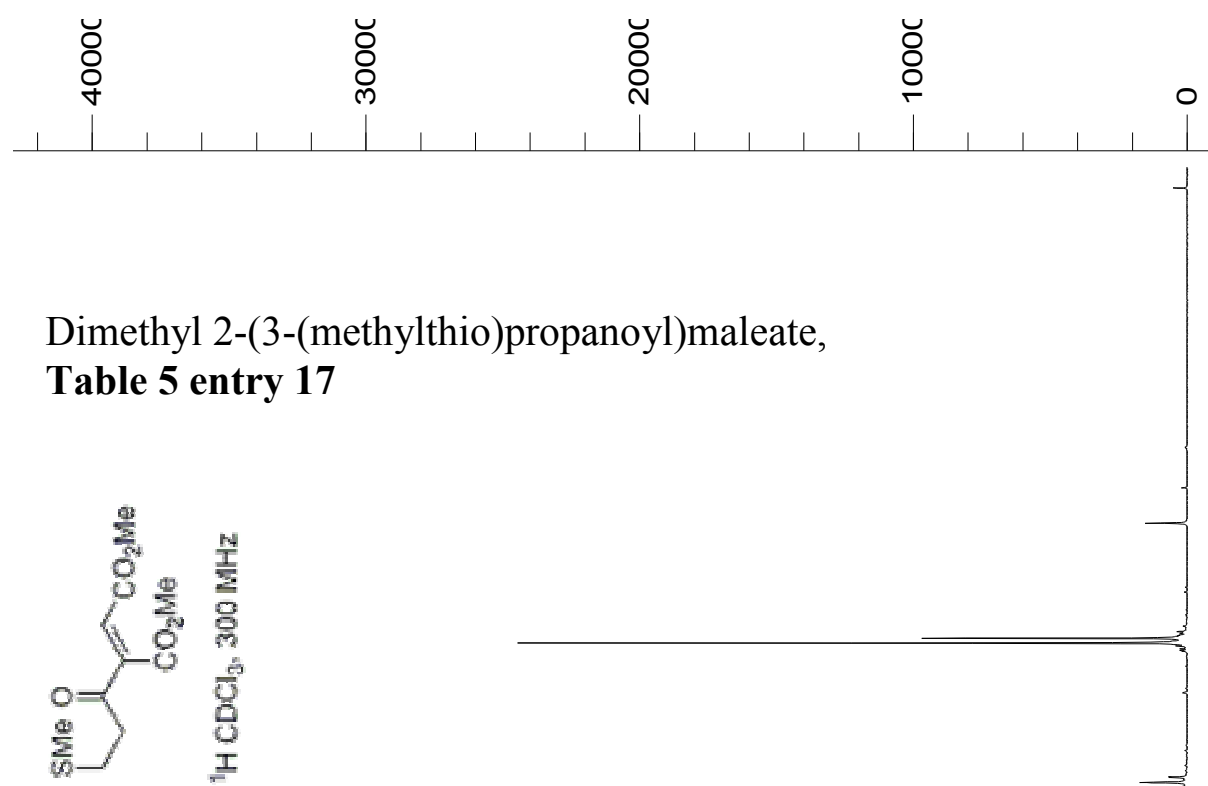

Dimethyl 2-(3-(methylthio)propanoyl)maleate,

Table 5 entry 17

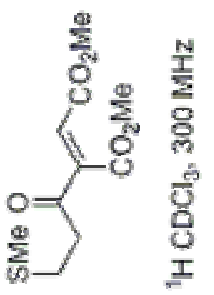




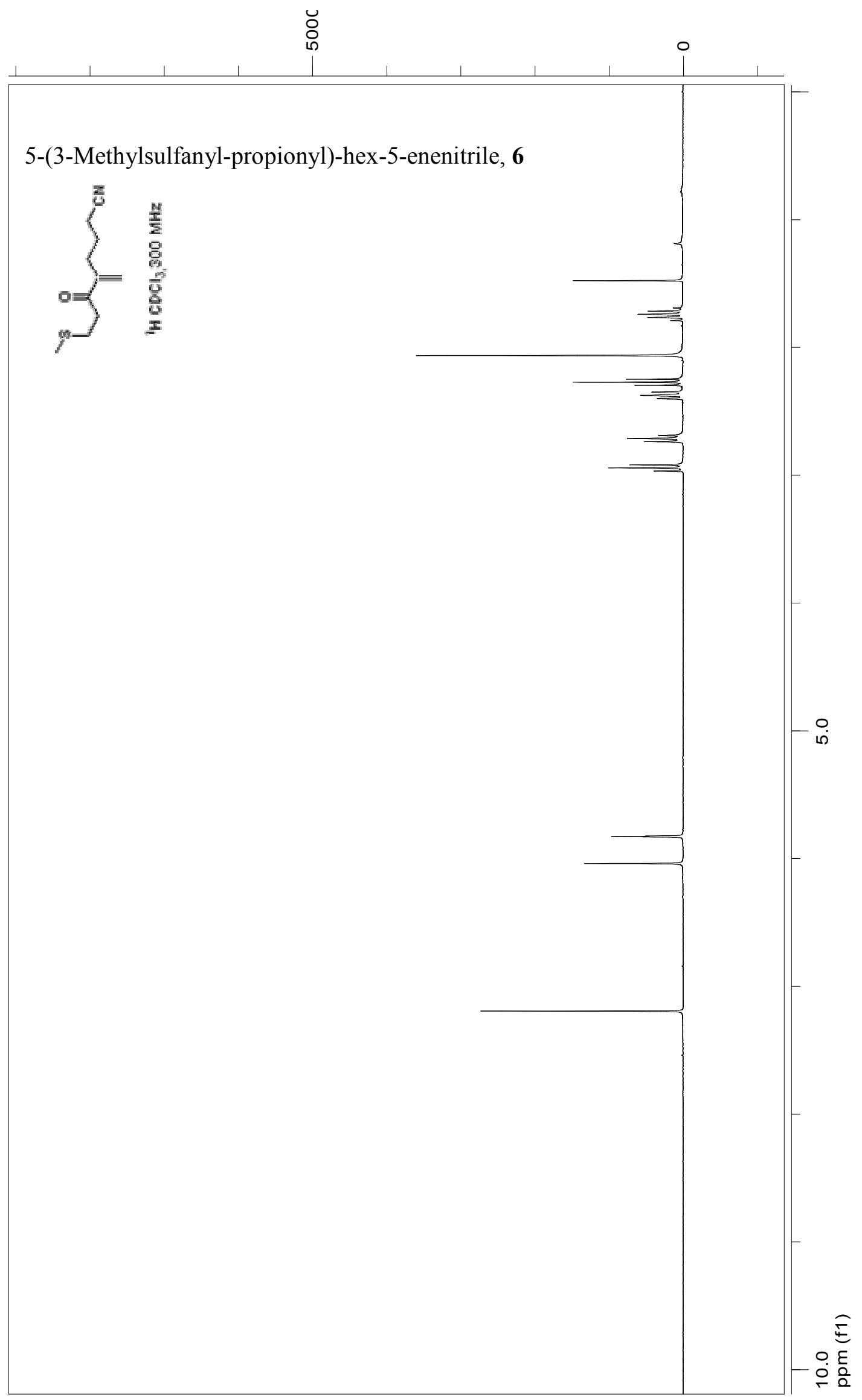




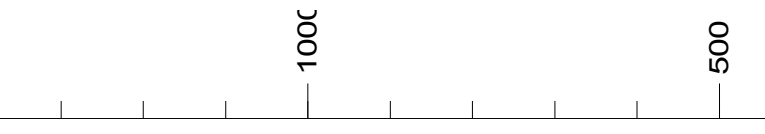

(E)-9-(methylthio)-7-oxonon-5-enenitrile, 7

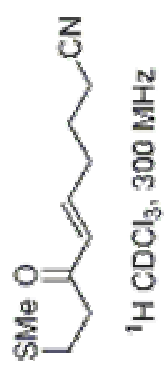




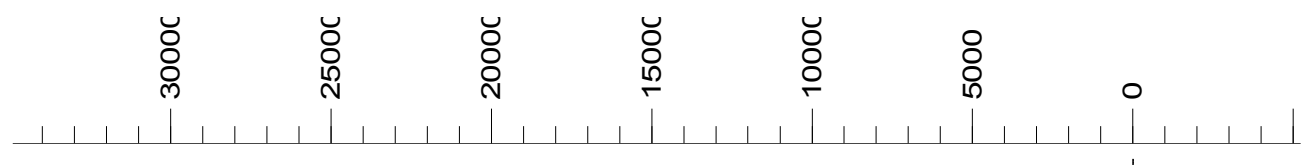

(E)-8-(methylthio)-6-oxooct-4-enenitrile, Table 6 entry 1

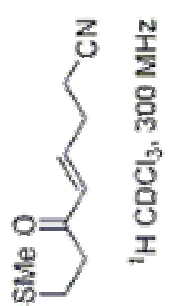




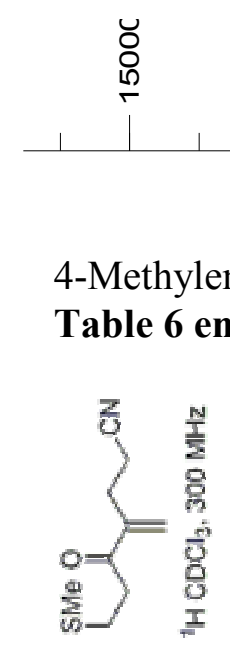

○)
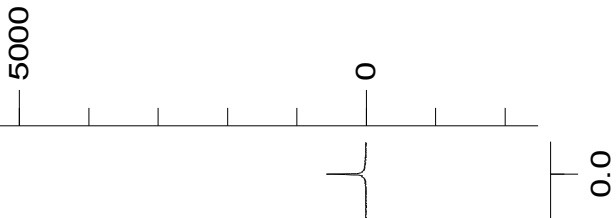

4-Methylene-7-(methylthio)-5-oxoheptanenitrile, 


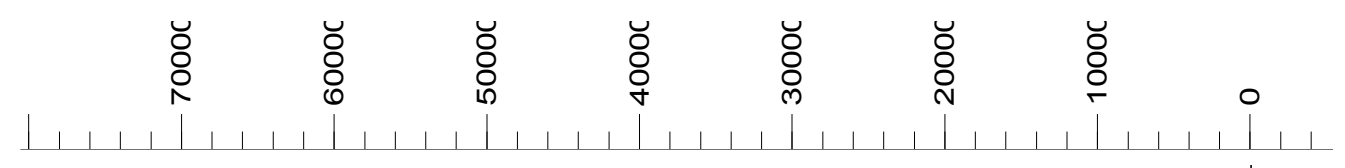

(E)-10-(methylthio)-8-oxodec-6-enenitrile, Table 6 entry 2

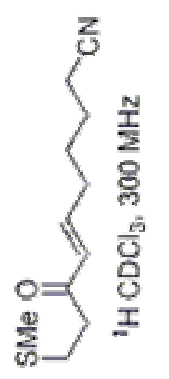



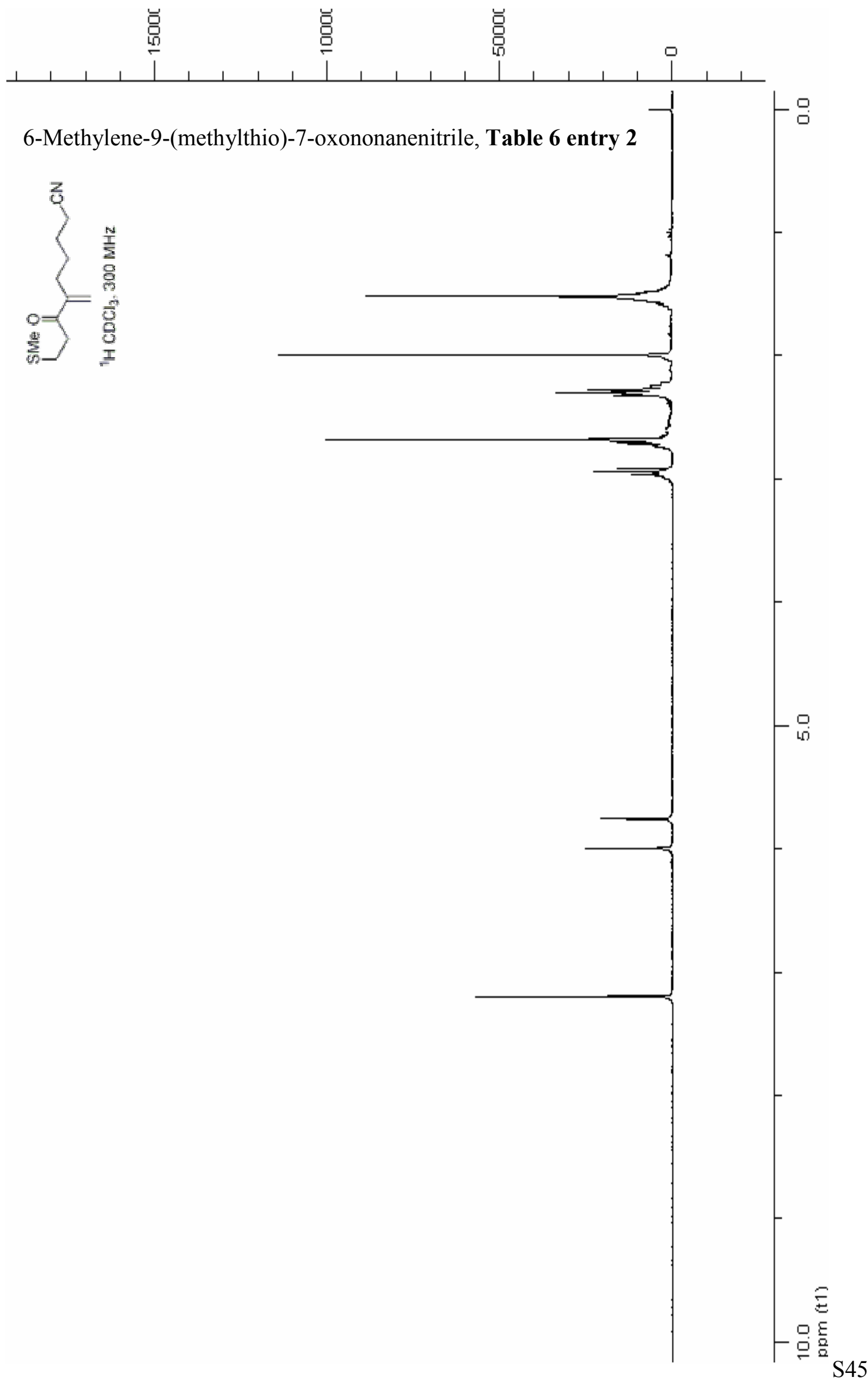


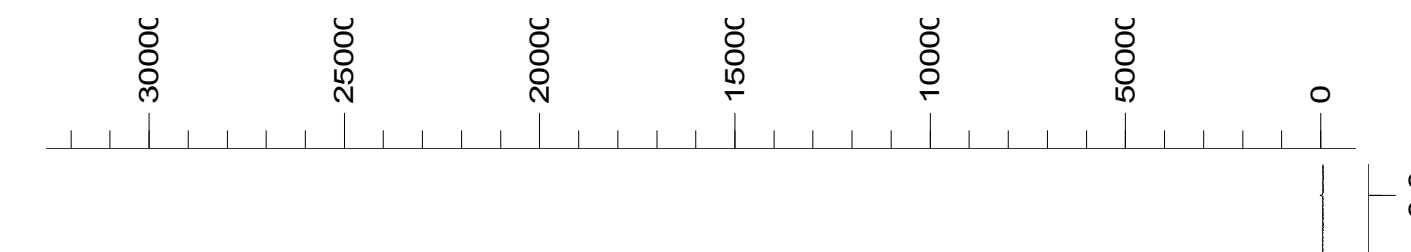

(E)-4-methyl-1-(methylthio)undec-4-en-3-one and (E)-4-ethylidene-1(methylthio)decan-3-one, Table 6 entry 3

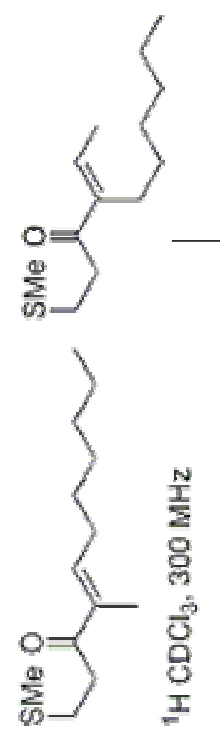




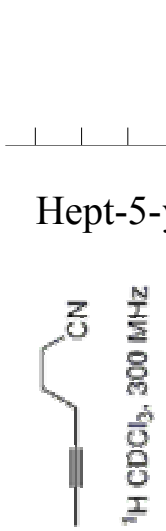

ర్

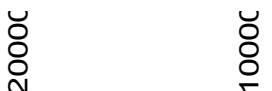

$\stackrel{\circ}{\circ}$

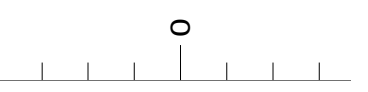

Hept-5-ynenitrile, Table 6 entry 4 substrate

$3 \frac{N}{\frac{N}{2}}$
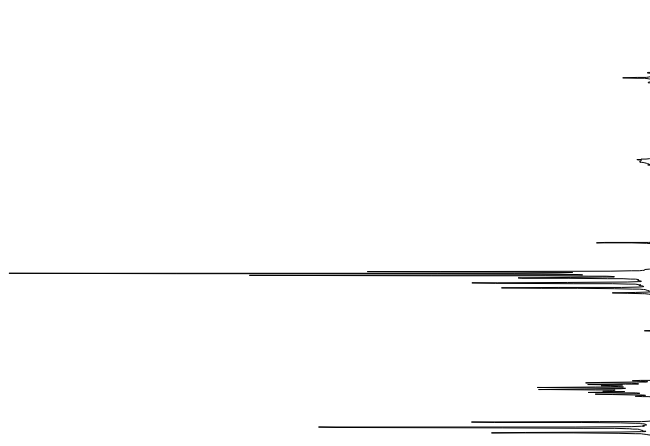


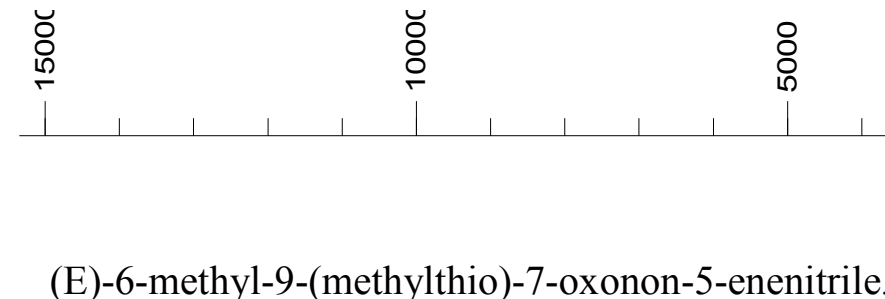

Table 6 entry 4

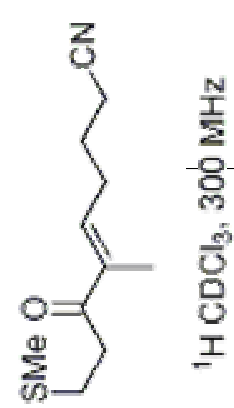



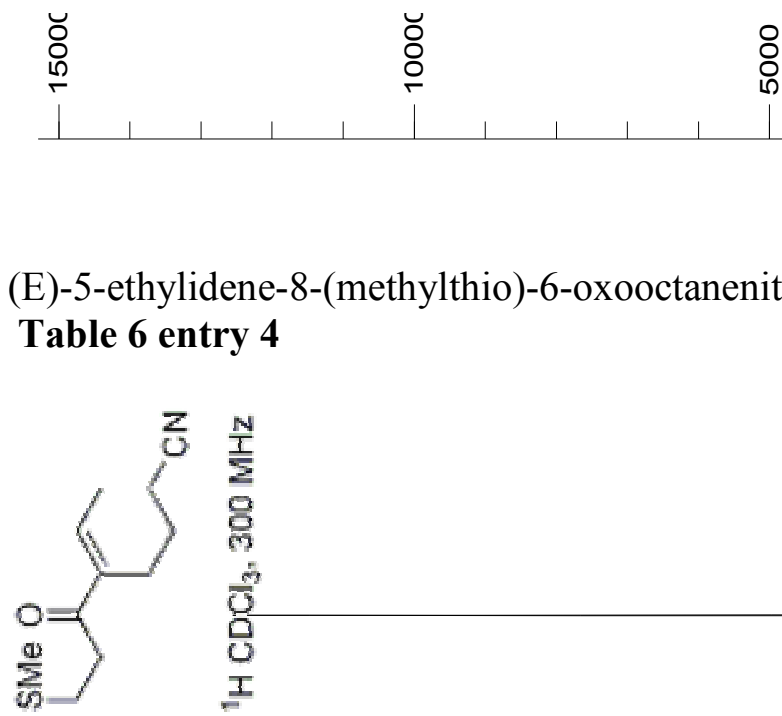

8

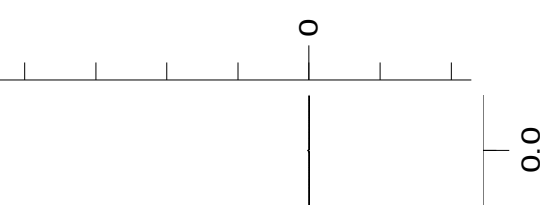

(E)-5-ethylidene-8-(methylthio)-6-oxooctanenitrile,

Table 6 entry 4

N
I
D
Dे
5
D
O
I 